\title{
Approximation by Linear Combinations of Fundamental Solution of Elliptic Systems of Partial Differential Operators
}

\author{
U. Hamann
}

Abstract. Let $\underline{L}(D)=\left(L_{i j}(D)\right)_{i, j=1, \ldots, N}$ be an elliptic system of partial differential operators of order $2 m, \underline{E}$ a fundamental solution of $\underline{L}$ and $\underline{E}$; the column vectors of $\underline{E}$. Let $\Gamma$ be the smooth boundary of a bounded domain $\Omega \subset R^{n}$ and $\underline{b}(x, D)=\left(b_{h j}(x, D)\right)_{\substack{n=1, \ldots, m \\ j=1, \ldots, N}}$ a normal system of boundary operators on $\Gamma$. We define $\underline{b}_{h} \underline{u}=\sum_{j=1}^{N} b_{h j} u_{j} \cdot\left(\underline{u}=\left(u_{1}, \ldots, u_{N}\right)^{T}\right)$. Furthermore let $\left(y_{k}\right)_{1}^{\infty} \subset R^{n} \backslash \bar{\Omega}$ be a sequence of points and $D_{h}(\Gamma)(h=1, \ldots, m)$ suitable function spaces over $\Gamma$ (e.g. $C^{\boldsymbol{s}}$-spaces or Sobolev spaces). It is investigated, under which conditions on the sequence $\left(y_{k}\right)_{1}^{\infty}$ the set

$$
\operatorname{span}\left\{\left(\left.\underline{b}_{1} D^{\alpha} \underline{E}_{j}\left(x-y_{k}\right)\right|_{x \in \Gamma}, \ldots,\left.\underline{b}_{m} D^{\alpha} \underline{E}_{j}\left(x-y_{k}\right)\right|_{x \in \Gamma}\right): k \in \mathbb{N} ;|\alpha| \geq 0 ; j=1, \ldots, N\right\}
$$

is dense in $\prod_{h=1}^{m} D_{h}(\Gamma)$.

Key words: Elliptic systems, fundamental solutions, approximations

AMS subject classification: $35 \mathrm{~A} 40,35 \mathrm{~J} 55$

\section{o. Introduction}

Let $\underline{L}(D)=\left(L_{i j}(D)\right)_{i, j=1, \ldots, N}$ be a Petrovskij-elliptic system of differential operators of order $2 m$ with constant coefficients and $\underline{E}=\left(E_{i j}\right)_{i, j=1, \ldots, N}$ a fundamental solution of $\underline{L}$. By $\underline{E}_{j}=\left(E_{1}, \ldots, E_{N j}\right)^{T}:(j=1, \ldots, N)$ we denote the column vectors of $\underline{E}$. Let $\dot{\Gamma}$ be the $C^{\infty}$-smooth boundary of a bounded domain $\Omega \subset R^{n}$ with. a connected complement $R^{n} \backslash \bar{\Omega}$. Furthermore let $\underline{b}(x, D)=\left(b_{h j}(x, D)\right)_{\substack{h=1, \ldots, m \\ j=1, \ldots, N}}$ be such a normal system of boundary operators on $\Gamma$ that $\underline{L}$ and $\underline{b}$ define an elliptic boundary value problem with respect to $\Omega$. The operators $\underline{b}_{h}(h=1, \ldots, m)$ are defined by $\underline{b}_{h} \underline{u}=\sum_{j=1}^{N} b_{h j} u_{j}\left(\underline{u}=\left(u_{1}, \ldots, u_{N}\right)^{T}\right)$. If $\left(y_{k}\right)_{1}^{\infty} \subset R^{n} \backslash \bar{\Omega}$ is a given sequence of points, let us consider finite linear combinations of vector functions of the form

U. Hamann: Universität Rostock, Fachbereich Mathematik, Universitätsplatz 1, D-18055 Rostock. 


$$
D^{\alpha} \underline{E}_{j}\left(x-y_{k}\right) \text { with } 1 \leq k<\infty, j=1, \ldots, N \text {, and } 0 \leq|\alpha|<\infty
$$

Let

$$
\underline{u}_{,, r}(x)=\sum_{k=1}^{l} \sum_{j=1}^{N} \sum_{|\alpha| \leq r} c_{k, j, \alpha} D^{\alpha} \underline{E}_{j}\left(x-y_{k}\right)
$$

be such a linear combination. A vector function

$$
\underline{f}=\left(f_{1}, \ldots, f_{m}\right)^{T} \text { with } f_{h} \in C^{s_{h}}(\Gamma)\left(s_{h} \geq 0, h=1, \ldots, m\right)
$$

is given on $\Gamma$. The problem consists in approximating the function $f$ by the $m$-tupel $\left(\underline{b}_{1} \underline{u}_{1, r}, \ldots, \underline{b}_{m} \underline{u}_{l, r}\right)^{T}$ in the space $\prod_{h=1}^{m} C^{s_{h}}(\Gamma)$. We will see that such an approximation is possible under certain conditions. These conditions essentially refer to the position of the points $y_{k}$. For instance, the following assertion holds:

We suppose that the sequence of points $\left(y_{k}\right)_{1}^{\infty} \subset R^{n} \backslash \bar{\Omega}$ is dense on a smooth $(n-1)$. dimensional surface $K \subset R^{n} \backslash \bar{\Omega}$. Then for each $f=\left(f_{1}, \ldots, f_{m}\right)^{T} \quad\left(f_{h} \in C^{*_{n}}(\Gamma), h=\right.$ $1, \ldots, m)$ and for all $\varepsilon>0$ there exists a natural number $l$ and coefficients $c_{k, j, \alpha} \quad(1 \leq$ $k \leq l, \quad|\alpha| \leq t-1, \cdot j=1, \ldots, N)$ with

$$
\sum_{h=1}^{m}\left\|\underline{b}_{h} \underline{u}_{h}-f_{h}\right\|_{C_{h(\Gamma)}}<\varepsilon
$$

for

$$
\underline{u}_{l}(x):=\sum_{k=1}^{l} \sum_{j=1}^{N} \sum_{|\alpha| \leq t-1} c_{k, j, \alpha} D^{\alpha} \underline{E}_{j}\left(x-y_{k}\right)
$$

if and only if the adjoint boundary value problem

$$
\underline{L}^{*} \underline{v}=\underline{Q} \quad \text { in } \Omega, \quad \underline{b}^{*} \underline{v}=\underline{0} \text { on } \Gamma
$$

has only the solution $\underline{v}=0$. Here $t$ is the maximal order of the $L_{i j}$. We note that the surface $K$ can be arbitrarily small.

This assertion is contained in Theorem 1 of the present paper. Other situations of the sequence of points $\left(y_{k}\right)_{1}^{\infty}$ will be investigated in the Theorems $2-6$. We will see that the construction of the vector function $\underline{u}_{y}$ depends on the position of the points $y_{k}$. Furthermore it will be shown that the functions $f_{h}$ can be elements of more general function spaces.

There exists a close relation between the present investigations and the approximation theorems of Beckert [1], and the developments of Beyer [2], Göpfert [5], Hamann [6], and Wildenhain .(15]. In these papers functions, given on a surface $\Gamma$, are approximated by solutions of boundary value problems of scalar elliptic differential operators with respect to a domain which involves $\Gamma$. This problem have been studied for Petrovskij-elliptic systems by Rojtberg and Sheftel [13]. They consider the case that $\underline{b}_{k}$ are Dirichlet boundary operators, and they need the supposition that the homogeneous Dirichlet problem for the adjoint system has at most one solution. 
Firstly Schulze and Wildenhain [14] got some results about the approximation of functions on $\Gamma$ by linear combinations of fundamental solutions. The Lamé equations were investigated by Beyer [3], Kupradze [10], Freeden and Reuter [4], and Hamann [8] with respect to the approximation problem considered in the present paper. In [4] and [10] the theoretical results were applied to numerical constructions. Here the approximation was considered in the norm of $\left(L_{2}(\Gamma)\right)^{3}$, and Beyer could prove in [3] that also each vector function from $\left(W_{2}^{*}(\Gamma)\right)^{3}$ with arbitrary integer $s \geq 0$ can be approximated in the norm of $\left(W_{2}^{*}(\Gamma)\right)^{3}$. In [8] it was shown that the approximation in the case of the Lame equations is possible in more general function spaces. In the present paper we generalize the results of [8] to systems which are elliptic in the sense of Petrovskij and to arbitrary normal boundary operators. Similar results were obtained by I. and Y.A. Rojtberg [11].

\section{Definitions and notations}

1.1 Let

$$
\begin{gathered}
\underline{L}(D)=\left(L_{i j}(D)\right)_{i, j=1, \ldots, N}, \quad L_{i j}(D)=\sum_{|\alpha| \leq t_{i j}} a_{\alpha}^{i j} D^{\alpha} \\
\left(\alpha=\left(\alpha_{1}, \ldots, \alpha_{n}\right), \quad D_{k}=\frac{1}{i} \frac{\partial}{\partial x_{k}}, \quad D^{\alpha}=D_{1}^{\alpha_{1}} \cdots D_{n}^{\alpha_{n}}\right)
\end{gathered}
$$

be a matrix of partial differential operators with constant coefficients. The operator $\underline{L}$ is said to be elliptic in the sense of Douglis-Nirenberg, if there exist integers $s_{1}, \ldots, s_{N}$ and $t_{1}, \ldots, t_{N}$ such that

$$
L_{i j}(D) \equiv 0 \text { for } s_{i}+t_{j}<0 \text { and } t_{i j} \leq s_{i}+t_{j} \text { for } s_{i}+t_{j} \geq 0
$$

while the characteristic polynomial

$$
l(\xi):=\operatorname{det}\left(L_{i j}^{0}(\xi)\right)_{i, j=1, \ldots, N} \neq 0
$$

for real $\underline{\xi}=\left(\xi_{1}, \ldots, \xi_{n}\right) \neq \underline{0}$. Here

$$
L_{i j}^{0}(\underline{\xi})=\left\{\begin{array}{cll}
\sum_{|\alpha|=s_{i}+t_{j}} a_{\alpha}^{i j} \underline{\xi}^{\alpha} & \text { for } & s_{i}+t_{j} \geq 0 \\
0 & \text { for } & s_{i}+t_{j}<0
\end{array}\right.
$$

and $\xi^{\alpha}=\xi_{1}^{\alpha_{1}} \cdots \xi_{n}^{\alpha_{n}}$.

If the operator is elliptic in the sense of Douglis-Nirenberg, then without loss of generality we may suppose that the numbers $s_{i}$ and $t_{j}$ are such that $\max \left\{s_{i}: 1 \leq i \leq N\right\}=0$. This follows from the fact that $s_{i}+t_{j}=\left(s_{i}-k\right)+\left(t_{j}+k\right)$. Then $\min \left\{t_{j}: 1 \leq j \leq N\right\} \geq 0$ and $t_{j}$ is the maximal order of differentiation of the function $u_{j}$ in $\underline{L}(D) \underline{u}$. By changing the enumeration of the functions and equations in $\underline{L}(D) \underline{u}$ we may always arrange that $t:=t_{1} \geq t_{2} \geq \cdots \geq t_{N} \geq 0$ and $0=s_{1} \geq s_{2} \geq \cdots \geq s_{N}$.

An operator elliptic in the sense of Douglis-Nirenberg is said to be elliptic in the sense of Petrovskij if $s_{1}=s_{2}=\cdots=s_{N}=0$. Let ord $\underline{L}:=\operatorname{ord} l(D)=\sum_{i=1}^{N} s_{i}+t_{i}$ be the order of $\underline{L}$.

\subsection{The operator}

$$
\underline{L}^{*}(D):=\left(L_{j i}^{*}(D)\right)_{i, j=1, \ldots, N}=\left(L_{i j}^{*}(D)\right)_{i, j=1, \ldots, N}^{T}, \quad L_{i j}^{*}(D)=\sum_{|\alpha| \leq t_{i j}}(-1)^{|\alpha|} a_{\alpha}^{i j} D^{\alpha}
$$


is the formally adjoint of $\underline{L}(D)$ in the sense of distribution theory. It is defined by

$$
\left\langle(\underline{L} \underline{\underline{u}})^{T}, \underline{v}\right\rangle:=\sum_{i=1}^{N} \sum_{j=1}^{N} \int_{R^{n}}\left(L_{i j} u_{j}\right) v_{i} d x=\sum_{j=1}^{N} \sum_{i=1}^{N} \int_{R^{n}} u_{j}\left(L_{i j}^{*} v_{i}\right) d x=\left\langle\underline{u}^{T}, \underline{L}^{*} \underline{v}\right\rangle
$$

for all $\underline{u}, \underline{v} \in\left(C_{0}^{\infty}\left(R^{n}\right)\right)^{N}$. It is clear that if the operator $\underline{L}$ is elliptic in the sense of Douglis-Nirenberg, then $\underline{L}^{*}$ is elliptic in the sense of Douglis-Nirenberg, too. For the formally adjoint system we have $t_{i j}^{*}=t_{j i} \leq s_{j}+t_{i}=s_{i}^{*}+t_{j}^{*}$, where $s_{i}^{*}=t_{i}-t$ and $t_{j}^{*}=s_{j}+t$.

1.3 Now we want to define the condition of Shapiro-Lopatinskij and the ellipticity of a boundary value problem. Let $\Gamma$ be the $C^{\infty}$-smooth boundary of a bounded domain $\Omega \subset R^{n}$. We suppose that the order of $\underline{L}$ is $2 \mathrm{~m}$. Let

$$
\underline{b}(x, D)=\left(b_{h j}(x, D)\right)_{\substack{h=1, \ldots, m \\ j=1, \ldots, N}}, \quad b_{h j}(x, D)=\sum_{|\alpha| \leq m_{h j}} b_{\alpha}^{h j}(x) D^{\alpha}, \quad b_{\alpha}^{h j} \in C^{\infty}(\Gamma)
$$

be a system of boundary operators on $\Gamma$. We define

$$
\begin{gathered}
r_{h}:=\max \left\{\left(m_{h j}-t_{j}\right): 1 \leq j \leq N\right\} \\
b_{h j}^{0}(\dot{x}, D) \doteq\left\{\begin{array}{ccc}
\sum_{|\alpha|=r_{h}+t_{j}} b_{\alpha}^{h j}(x) D^{\alpha} & \text { for } & r_{h}+t_{j} \geq 0 \\
0 & \text { for } & r_{h}+t_{j}<0,
\end{array}\right.
\end{gathered}
$$

and $\underline{b}^{0}(x, D)=\left(b_{h j}^{0}(x, D)_{\substack{h=1, \ldots, m \\ j=1, \ldots, N}}\right.$. For a fixed point $x_{0} \in \Gamma$ we consider the initial value problem

$$
\begin{aligned}
& \underline{L}^{0}\left(\underline{\xi^{\prime}}, \frac{1}{i} \frac{d}{d t}\right) \underline{v}(t)=\underline{0} \text { for } t>0 \\
& \underline{b}^{0}\left(x_{0} ; \underline{\xi}^{\prime}, \frac{1}{i} \frac{d}{d t}\right) \underline{v}(0)=\underline{h} \in C^{m} .
\end{aligned}
$$

Here we have replaced $D=\left(D_{1}, \cdots, D_{n}\right)$ by $\left(\xi_{1}, \cdots, \xi_{n-1}, \frac{1}{i} \frac{d}{d t}\right)\left(\xi^{\prime}=\left(\xi_{1}, \cdots, \xi_{n-1}\right)\right)$. Furthermore $\underline{v}=\left(v_{1}, \ldots, v_{N}\right)^{T}$ is a vector function on $R_{+}^{1}=\left\{t \in R^{1}: t \geq 0\right\}$. Let $\mathcal{M}^{+}$be the set of all solutions $\underline{v}$ of problem $(1),(2)$ with $\lim _{t \rightarrow+\infty} \underline{v}(t)=\underline{0}$.

One says that the Shapiro-Lopatinskij condition is satisfied in the point $x_{0} \in \Gamma$, if the problem (1),(2) has a unique solution $\underline{v} \in \mathcal{M}^{+}$for every vector $\xi^{\prime} \in R^{n-1}$ with $\xi^{\prime} \neq \underline{0}$ and for every $\underline{h}=\left(h_{1}, \ldots, h_{m}\right)^{T} \in C^{m}$.

Furthermore the boundary value problem defined by $\underline{L}$ and $\underline{b}$ is said to be elliptic in $\bar{\Omega}$, if the following two properties are fulfilled:

1. $L$ is elliptic in the sense of Douglis-Nirenberg.

2. In every boundary point $x_{0} \in \Gamma$ the condition of Shapiro-Lopatinskij is satisfied.

1.4 Now we want to define normal boundary conditions and the Green formula. Let $t_{1}, \ldots, t_{N}$ be integers with $t:=t_{1} \geq t_{2} \geq \cdots t_{N} \geq 0$. We set $T=\left(t_{1}, \ldots, t_{N}\right)$ and $|T|=\sum_{j=1}^{N} t_{j}$. We denote by $N_{s}(s=1, \ldots, t)$ the number of those values of $j$ for which $t_{j} \geq s$. It is easy to see that $\sum_{s=1}^{t} N_{s}=\sum_{j=1}^{N} t_{j}=|T|$ and $N \geq N_{1} \geq \cdots \geq N_{t}$. We put also $N_{s}^{\prime}=N_{t-s+1}(s=1, \ldots, t)$ and note that $j \leq N_{,}^{\prime}$ if and only if $t-t_{j} \leq s-1$. 
Definition 1. A Dirichlet matrix on $\Gamma$ of order $T=\left(t_{1}, \ldots, t_{N}\right)$ is a matrix with $|T|$ rows, which may be reduced by permutaion of rows to the form

$$
\underline{B}=\underline{B}(x, D)=\left(\begin{array}{c}
\underline{B}^{1} \\
\vdots \\
\underline{B}^{t}
\end{array}\right), \quad \underline{B}^{s}=\left(B_{k j}^{s}(x, D)\right)_{\substack{k=1, \ldots, N^{\prime} \\
j=1, \ldots, N}} \quad(s=1, \ldots, t)
$$

where

$$
B_{k j}^{o}(x, D)=\left\{\begin{array}{cll}
\sum_{|\alpha| \leq s-1-\left(t-t_{j}\right)} B_{k j}^{e a}(x) D^{\alpha} & \text { for } & j=1, \ldots, N^{\prime} \\
0 & \text { for } & j=N_{s+1}^{\prime}, \ldots, N
\end{array}\right.
$$

while

$$
\operatorname{det}\left(B_{k j}^{0 s}\left(x, \eta_{x}\right)\right)_{k, j=1, \ldots, N:} \neq 0
$$

at every point $x \in \Gamma$. Here $B_{k j}^{0 a}(x, D)=\sum_{|\alpha|=s-1-\left(t-t_{j}\right)} B_{k j}^{s \alpha}(x) D^{\alpha}$ and $\eta_{x}$ is the unit nor$\mathrm{mal}$ to $\Gamma$ at $x$.

Definition 2. The matrix $\underline{b}(x, D)=\left(b_{h j}(x, D)\right)_{\substack{h=1, \ldots, m \\ j=1, \ldots, N}}$ will said to be $T$-normal if it may be supplemented by new rows to a Dirichlet matrix on $\Gamma$ of order $T$. We shall call it locally $T$-normal if for each point $x_{0} \in \Gamma$ there exists a neighbourhood $U\left(x_{0}\right)$ in $\Gamma$ in which the matrix $\underline{b}(x, D)$ may be supplemented by new rows to a Dirichlet matrix of order $T$ :

For the multi-index $Q=\left(q_{1}, \ldots, q_{N}\right)\left(q_{k}\right.$ integers) we define $W_{p}^{Q}(\Omega)=W_{p}^{q_{1}}(\Omega) \times \cdots \times$ $W_{p}^{q_{N}}(\Omega)\left(W_{p}^{q_{k}}(\Omega)\right.$ Sobolev spaces) and $C^{Q}(\bar{\Omega})=C^{q_{1}}(\bar{\Omega}) \times \cdots \times C^{q_{N}}(\bar{\Omega})$ for $q_{k} \geq 0$. For an integer $k_{0}$ we put $k_{0}+Q=\left(k_{0}+q_{1}, \ldots, k_{0}+q_{N}\right)$.

The following both lemmas were proved by Rojtberg and Sheftel [12].

Lemma 1. Suppose that the operator $\underline{L}(D)$ of order $2 m$ is elliptic in the sense of Petrovskij (ord $\left.L_{i j}(D) \leq t_{j} \quad\left(j=1, \ldots, N ; \quad s_{1}=\cdots=s_{N}=0, \quad|T|=2 m\right)\right)$. Suppose that the matrix $\underline{b}(x, D)=\left(b_{h j}(x, D)\right)_{\substack{n=1, \ldots, \ldots \\ j=1, \ldots, N}}$ of boundary conditiors is T-normal. Then there exists a $T$-normal matrix $\underline{c}(x, D)$ which completes $\underline{b}(x, D)$ to a Dirichlet matrix of order $T=\left(t_{1}, \ldots, t_{N}\right)$ on $\Gamma$. If $\underline{c}(x, D)$ is fixed, then there exist matrices

$$
\underline{b}^{*}(x, D)=\left(b_{h j}^{*}(x, D)\right)_{\substack{h=1, \ldots, m \\ j=1, \ldots, N}} \text { and } \underline{c}^{*}(x, D)=\left(c_{h j}^{*}(x, D)\right)_{\substack{n=1, \ldots, m \\ j=1, \ldots, N}}
$$

of boundary operators with coefficients from $C^{\infty}(\Gamma)$ and with the following properties:

(i) $\underline{b}^{*}$ and $\underline{c}^{*}$ are locally $T^{\prime}$-normal with $T^{\prime}=(t, t, \ldots, t)$.

(ii) There exist negative integers $r_{h}, r_{h}^{c}, r_{h}^{*}, r_{h}^{c^{*}}(h=1, \ldots, m)$ such that ord $b_{h j} \leq$ $r_{h}+t_{j}$, ord $c_{h j} \leq r_{h}^{c}+t_{j}$, ord $b_{h j}^{*} \leq r_{h}^{*}+t$, ord $c_{h j}^{*} \leq r_{h}^{c^{*}}+t$, while in each of these inequalities there is for each $h$ at least one $j$ for which the equality sign holds. If now $r_{h}+t_{j}<0$, then $b_{h j}(x, D) \equiv 0$, if $r_{h}^{c}+t_{j}<0$, then $c_{h j}(x, D) \equiv 0$, and so forth. In addition, $-r_{h}-r_{h}^{c^{*}}=-r_{h}^{c}-r_{h}^{*}=t+1$ for $h=1, \ldots, m$.

(iii) For all $\underline{u} \in C^{T}(\bar{\Omega}), \underline{v} \in C^{T^{\prime}}(\bar{\Omega}) \quad\left(T^{\prime}=(t, t, \ldots, t)\right)$ the Green formula

$$
\sum_{i=1}^{N} \int_{\Omega}\left(\sum_{j=1}^{N} L_{i j} u_{j}\right) v_{i} d x+\sum_{h=1}^{m} \int_{\Gamma}\left(\sum_{j=1}^{N} b_{h j} u_{j}\right)\left(\sum_{i=1}^{N} c_{h i}^{*} v_{i}\right) d \sigma
$$




$$
=\sum_{j=1}^{N} \int_{\Omega} u_{j}\left(\sum_{i=1}^{N} L_{i j}^{*} v_{i}\right) d x+\sum_{h=1}^{m} \int_{\Gamma}\left(\sum_{j=1}^{N} c_{h}, u_{j}\right)\left(\sum_{i=1}^{N} b_{h i}^{*} v_{i}\right) d \sigma
$$

or shorter

$$
\left\langle(\underline{L} \underline{u})^{T}, \underline{v}\right\rangle+\sum_{h=1}^{m}\left\langle\underline{b}_{h} \underline{u}, \underline{c}_{h}^{*} \underline{v}\right\rangle=\left\langle\left(\underline{u}^{T}\right), \underline{L}^{*} \underline{v}\right\rangle+\sum_{h=1}^{m}\left\langle\underline{c}_{h} \underline{u}, \underline{b}_{h} \underline{v}\right\rangle
$$

holds. Here $\underline{b}_{h} \underline{u}=\sum_{j=1}^{N} b_{h j} u_{j}$, the meaning of $\underline{c}_{h}^{*} \underline{v}, \underline{c}_{h} \underline{u}, \underline{b}_{h}^{*} \underline{v}$ is analogous.

If the system $\underline{L}$ is elliptic in the sense of Petrovskij, if $\underline{b}$ is a $T$-normal system, and if the boundary value problem defined by $\underline{L}$ and $\underline{b}$ is elliptic, then the boundary value problem defined by $\underline{L}^{*}$ and $\underline{b}^{*}$ is elliptic as well (see [12: Theorem 2]). But we note that the formally adjoint system $\underline{L}^{*}$ is in general elliptic only in the sense of Douglis-Nirenberg.

Let

$$
N^{*}:=\left\{\underline{v} \in\left(C^{\infty}(\bar{\Omega})\right)^{N}: \underline{L}^{*} \underline{v}=\underline{0} \text { in } \Omega, \quad \underline{b}^{*} \underline{v}=\underline{0} \text { on } \Gamma\right\}
$$

be the finite-dimensional kernel of the operator $\left\{\underline{L}^{*}, \underline{b}^{*}\right\}$.

Lemma 2. Suppose that the system $\underline{L}$ is elliptic in the sense of Petrovskij, the boundary conditions $\underline{b}$ are $T$-normal, and that $\underline{L}$ and $\underline{b}$ define an elliptic boundary value problem. Then the problem $\underline{L} \underline{u}=\underline{f}$ in $\Omega, \underline{b} \underline{u}=\underline{0}$ on $\Gamma$ with $f \in\left(C^{\infty}(\bar{\Omega})\right)^{N}$ has a solution if and only if $\left\langle\underline{f}^{T}, \underline{v}\right):=\sum_{i=1}^{N} \int_{\Omega} f_{i} v_{i} d x=0$ for all $\underline{v}=\left(v_{1}, \ldots, v_{N}\right)^{T} \in N^{*}$. The solution $\underline{u}$ belongs to $\left(C^{\infty}(\bar{\Omega})^{N}\right.$.

1.5 Let $\underline{E}=\left(E_{i j}\right)_{i, j=1, \ldots, N}$ be a fundamental solution of $\underline{L}$, i.e.,

$$
\sum_{i=1}^{N} L_{k i} E_{i j}=\left\{\begin{array}{lll}
\delta & \text { for } & k=j \\
0 & \text { for } & k \neq j
\end{array} \quad(k, j=1, \ldots, N ; \delta \text { the Dirac measure })\right.
$$

We denote by $\underline{E}_{j}=\left(E_{1 j}, \ldots, E_{N j}\right)^{T}(j=1, \ldots, N)$ the columus of the fundamental solution $\underline{E}$. The matrix function $\underline{E}^{*}$ defined by $\underline{E}^{*}(x)=(\underline{E}(-x))^{T}$ is a fundamental solution of $\underline{L}^{*}$.

1.6 If $X$ is a normed space we denote by $X^{\prime}$ its dual space and by $\langle f, F\rangle$ the pairing between an element $f$ of $X$ and $F$ of $X^{\prime}$.

For normed spaces $X, Y$ let $L(X, Y)$ be the set of all continuous and linear operators from $X$ into $Y$. For $B \in L(X, Y)$ let $B^{\prime} \in L\left(Y^{\prime}, X^{\prime}\right)$ be the dual operator defined by the equation $\langle B f, F\rangle=\left\langle f, B^{\prime} F\right\rangle$ for each $f \in X$ and for each $F \in X^{\prime}$. Furthermore we denote by $(f, F)$ also the pairing between an element $f$ of $C_{0}^{\infty}\left(R^{n}\right)$ and a distribution $F$ of $D^{\prime}\left(R^{n}\right)$.

1.7 Let $X_{1}, \ldots, X_{N}$ be normed spaces and $X_{1}^{\prime}, \ldots, X_{N}^{\prime}$ their dual spaces. For $g=$ $\left(g_{1}, \ldots, g_{N}\right)^{T}, g_{j} \in X_{j}^{\prime}(j=1, \ldots, N)$, and $f=\left(f_{1}, \ldots, f_{N}\right)^{T}, f_{j} \in X_{j}(j=1, \ldots, N)$ we define

$$
\left\langle\underline{f}^{T}, g\right)=\sum_{j=1}^{N}\left(f_{j}, g_{j}\right)
$$


For a matrix $\underline{\Phi}=\left(\varphi_{i j}\right)_{\substack{i=1, \ldots, q \\ j=1, \ldots, N}}, \varphi_{i j} \in X_{j}(i=1, \ldots, q ; j=1, \ldots, N)$ let

$$
\langle\underline{\Phi}, g\rangle=\sum_{j=1}^{N}\left(\begin{array}{c}
\left\langle\varphi_{1 j}, g_{j}\right\rangle \\
\left\langle\varphi_{2 j}, g_{j}\right\rangle \\
\vdots \\
\left\langle\varphi_{q j}, g_{j}\right\rangle
\end{array}\right)
$$

For $g_{j} \in D^{\prime}\left(R^{n}\right)(j=1, \ldots, N)$ and $\varphi_{i j} \in C_{0}^{\infty}\left(R^{n}\right)(i=1, \ldots, q ; j=1, \ldots, N)$ we define the convolution

$$
(\underline{\Phi} * \underline{g})(y)=\sum_{j=1}^{N}\left(\begin{array}{c}
\left(\varphi_{1 j} * g_{j}\right)(y) \\
\left(\varphi_{2 j} * g_{j}\right)(y) \\
\vdots \\
\left(\varphi_{q j} * g_{j}\right)(y)
\end{array}\right)=\langle\underline{\Phi}(y-x), \underline{g}(x)\rangle=\sum_{j=1}^{N}\left(\begin{array}{c}
\left\langle\varphi_{1 j}(y-x), g_{j}(x)\right\rangle \\
\left\langle\varphi_{2 j}(y-x), g_{j}(x)\right\rangle \\
\vdots \\
\vdots \\
\left\langle\varphi_{q j}(y-x), g_{j}(x)\right\rangle
\end{array}\right)
$$

If the $g_{j}(j=1, \ldots, N)$ are distributions with compact supports and $\varphi_{i j} \in D^{\prime}\left(R^{n}\right)$ $(i=1, \ldots, q ; j=1, \ldots, N)$ we define

$$
\Phi * g=\sum_{j=1}^{N}\left(\begin{array}{c}
\varphi_{1 j} * g_{j} \\
\varphi_{2 j} * g_{j} \\
\vdots \\
\varphi_{q j} * g_{j}
\end{array}\right)
$$

If $\underline{\Phi}=\underline{E}$ is the fundamental solution of $\underline{L}$ we get

$$
\underline{L}(\underline{E} * g)=\underline{g}
$$

This follows from the equation

$$
\underline{L}\left(\begin{array}{c}
E_{11} * g_{1} \\
E_{21} * g_{1} \\
\vdots \\
E_{N 1} * g_{1}
\end{array}\right)=\sum_{j=1}^{N}\left(\begin{array}{c}
L_{1 j}\left(E_{j 1} * g_{1}\right) \\
L_{2 j}\left(E_{j 1} * g_{1}\right) \\
\vdots \\
L_{N j}\left(E_{j 1} * g_{1}\right)
\end{array}\right)=\left(\begin{array}{c}
\delta * g_{1} \\
0 \\
\vdots \\
0
\end{array}\right)=\left(\begin{array}{c}
g_{1} \\
0 \\
\vdots \\
0
\end{array}\right)
$$

and similar considerations for $g_{2}, \ldots, g_{N}$.

1.8 The spaces of functions on $\Gamma$ (the smooth boundary of a domain $\Omega \subset R^{n}$ ) we are going to approximate are Banach spaces with special properties. Now we formulate some conditions for a Banach space $D(\Gamma)$.

(a1) There exists an integer $s_{0} \geq 0$ so that $C^{\nu_{0}}(\Gamma)$ is continuously imbedded in $D(\Gamma)$.

(a2) It holds $\overline{C^{\infty}(\Gamma)}=D(\Gamma)$ (closure in $D(\Gamma)$ ).

(a3) If $\psi_{0} \in C^{\infty}(\Gamma)$, then $\left|\int_{\Gamma} \varphi \psi_{0} d \sigma\right| \leq C\|\varphi\|_{D(\Gamma)}\left(\varphi \in C^{\infty}(\Gamma) ; C=C\left(\psi_{0}\right)\right.$ a constant $)$.

Let $D_{1}(\Gamma), \ldots, D_{m}(\Gamma)$ be Banach spaces with properties (a1), (a2) and (a3). Because of property (a3) there exists for every $\psi_{0} \in C^{\infty}(\Gamma)$ and for every $h(1 \leq h \leq m)$ a uniquely (because of the property (a2)) determined functional $F_{h, \psi_{0}} \in\left(D_{h}(\Gamma)\right)^{\prime}$ with

$$
\left(\varphi, F_{h, \psi_{0}}\right)=\int_{\Gamma} \varphi \psi_{0} d \sigma
$$


for all $\varphi \in C^{\infty}(\Gamma)$

If now $\underline{v} \in\left(C^{\infty}(\bar{\Omega})\right)^{N}$ is an arbitrary, but fixed vector function, so we can define by

$$
\left\langle\varphi^{T}, \underline{c}^{*} \underline{v}\right\rangle=\sum_{h=1}^{m} \int_{\Gamma} \varphi_{h} \underline{c}_{h}^{*} \underline{v} d \sigma \quad \text { for all } \quad \varphi \in\left(C^{\infty}(\Gamma)\right)^{N}
$$

$\left(\underline{c}_{h}^{*} \underline{v}=\sum_{j=1}^{N} c_{h j}^{*} v_{j}, \quad c_{h j}^{*}\right.$ from Green's formula) an element from $\left(\prod_{h=1}^{m} D_{h}(\Gamma)\right)^{\prime}$, which we

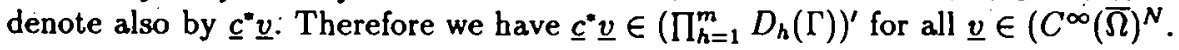

\section{Results}

Firstly we want to summarize the suppositions.

1. Let $\Gamma$ be the $C^{\infty}$-smooth boundary of a bounded domain $\Omega \subset R^{n}$ with a connected complement $R^{n} \backslash \bar{\Omega}$.

2. Let $\underline{L}(D)=\left(L_{i j}(D)\right)_{i, j=1, \ldots, N}$ be a Petrovskij-elliptic operator of order $2 m$ with ord $L_{i j} \leq t_{j}\left(i, j=1, \ldots, N ; \quad t:=t_{1} \geq t_{2} \geq \cdots \geq t_{N}\right)$ and with constant coefficients. Furthermore let $\underline{b}(x, D)=\left(b_{h j}(x, D)\right)_{\substack{h=1, \ldots, m \\ j=1, \ldots, N}}$ be a $T$-normal system $\left(T=\left(t_{1}, \ldots, t_{N}\right)\right)$ of boundary operators on $\Gamma$ with ord $b_{h j} \leq r_{h}+t_{j}\left(R=\left(r_{1}, \ldots, r_{m}\right), \quad r_{h} \leq-1\right)$ and with $C^{\infty}$-coefficients. We assume that the boundary problem defined by $\underline{L}$ and $\underline{b}$ is elliptic. Let $t=t_{1}$ be the maximal order of the $L_{i j}$.

3. We denote by $\underline{E}=\left(E_{i j}\right)_{i, j=1, \ldots, N}$ the fundamental solution of $\underline{L}$. The columns of $\underline{E}$ are denoted by $\underline{E}_{j}$.

4. The Banach spaces $D_{1}(\Gamma), \ldots, D_{m}(\Gamma)$ have the properties (a1) and (a2). If $N^{*} \neq$ $\{\underline{0}\}$, we additionally require the property (a3).

Some examples for such spaces are the spaces $C^{s}(\Gamma)$ for arbitrary integers $s \geq 0$, Sobolev-Slobodeckij spaces $W_{p}^{\prime}(\Gamma)$ for arbitrary real numbers $0 \leq s<\infty, 1 \leq p<\infty$, and the dual spaces $\left(W_{p}^{\prime}(\Gamma)\right)^{\prime}$ of $W_{p}^{\prime}(\Gamma)$ for $0 \leq s<\infty, 1<p<\infty$. The Hölder spaces $C^{s, a}(\Gamma) \quad(0 \leq s<\infty$ integer, $0<a \leq 1)$ do not satisfy the condition (a2).

Theorem 1. Let $K \subset R^{n} \backslash \bar{\Omega}$ be a $C^{1}$-smooth $(n-1)$-dimensional compact surface. We suppose that the sequence $\left(y_{k}\right)_{1}^{\infty} \subset R^{n} \backslash \bar{\Omega}$ is dense on $K$. Then for a vector function $\frac{f}{1}=\left(f_{h}\right)_{1}^{m} \in \prod_{h=1}^{m} D_{h}(\Gamma)$ there exist numbers $c_{k, j, a}^{(l)}(1 \leq l<\infty ; 1 \leq k \leq l ; j=$ $1, \ldots, N ;|\alpha| \leq t-1)$ such that

$$
\lim _{l \rightarrow \infty} \sum_{h=1}^{m}\left\|f_{h}-\underline{b}_{h} \underline{u}_{h}\right\|_{D_{h}(\Gamma)}=0
$$

holds for

$$
\underline{u}_{l}(x)=\sum_{k=1}^{l} \sum_{j=1}^{N} \sum_{|\alpha| \leq t-1} c_{k, j, \alpha}^{(l)} D^{\alpha} \underline{E}_{j}\left(x-\dot{y}_{k}\right)
$$

if and only if the condition

$$
\left\langle\underline{f}^{T} ; \underline{c}^{\bullet} \underline{v}\right\rangle=0
$$

is satisfied for all $\underline{v} \in N^{*}$. 
Remarks. 1. The requirement of $C^{1}$-smoothness of the compact set $K$ can be weakened (cf. Lemma 13 and [7: Satz l]). The essential property of $K$ is only the $(n-1)$-dimensionality. 2. The points $y_{k}$ can lie on $K$, but this is not necessary. 3 . If $R^{n} \backslash \bar{\Omega}$ consists of more than one connected component, then the assertion of Theorem 1 is also valid, if in each connected component lies such a compact set $K$. Then the sequence $\left(y_{k}\right)_{1}^{\infty}$ must be dense in the union of these $K$. 4. Since $N^{*}$ is finite-dimensional the supposition (3) contains only a finite number of conditions, in the case $N^{*}=\{0 \underline{\text { even }}$ no condition. 5. If the functions $f_{h}$ are integrable, then $\left\langle\underline{f}^{T}, \underline{c}^{*} \underline{v}\right\rangle=\sum_{h=1}^{m} \int_{\Gamma} f_{h} \underline{c}_{h}^{*} \underline{v} d \sigma$. 6. The condition (3) is also the solvability condition of the boundary value problem $\underline{L} \underline{u}=\underline{0}$ in $\Omega, \quad \underline{b} \underline{v}=\underline{f}$ on $\Gamma$, if the $f_{h}$ are sufficiently often differentiable. 7 . Indeed, the boundary operators $\underline{b}^{*}$ and $\underline{c}^{*}$ depend on the special choice of the system $\underline{c}$ in Lemma 1 , but the.condition (3) is independent of the choice of the system $\underline{c}$ (see $i 6: \dot{p}$. 300$]$ ).

Theorem 2. Let $\tilde{T}=\left(\tilde{t}_{1}, \ldots, \tilde{t}_{N}\right)$ be a multi-index with $0 \leq \tilde{t}_{j} \leq t$ and $\sum_{j=1}^{N} \tilde{t}_{j}=m$. Further let $\partial U$ be the boundary of a bounded open set $U \subset \bar{U} \subset R^{n} \backslash \bar{\Omega}$, for which the Dirichlet problem

$$
\underline{L}^{*} \underline{v}=\underline{0} \quad \text { in } U, \quad D^{\alpha} v_{j}=0 \text { on } \partial U \text { for }|\alpha| \leq \tilde{t}_{j}-1 \quad(j=1, \ldots, m)
$$

has in $\left(C^{\infty}(\bar{U})\right)^{N}$ only the trivial solution. (If $\tilde{t}_{j}=0$, then $D^{\alpha} v_{j}=0$ on $\partial U$ for $|\alpha| \leq \tilde{t}_{j}-1$ is no condition on $v_{j}$.) If the sequence $\left(y_{k}\right)_{1}^{\infty} \subset R^{n} \backslash \bar{\Omega}$ is dense on $\partial U$, then the assertion of Theorem 1 holds with

$$
\underline{u}_{1}(x)=\sum_{k=1}^{l} \sum_{j=1}^{N} \sum_{|\alpha| \leq \bar{i}_{j}-1} c_{k, j, \alpha}^{(l)} D^{\alpha} \underline{E}_{j}\left(x-y_{k}\right) .
$$

As distinct from Theorem 1 here we need less derivatives of $\underline{E}_{j}$.

Theorem 3. Let $y_{0}=\left(y_{0,1}, y_{0,2}, \ldots, y_{0, n}\right) \in R^{n} \backslash \bar{\Omega}$ be any point. Furthermore for $i=$ $1, \ldots, n$ let $\left(x_{i}^{(l)}\right)_{l=1}^{\infty} \subset R^{1} ; x_{i}^{(l)} \neq y_{0, i}$ for each $l$, be a real sequence with $y_{0, i}$ as accumulation point, such that $\left(x_{1}^{\left(l_{1}\right)}, \ldots, x_{n}^{\left(l_{n}\right)}\right) \in R^{n} \backslash \bar{\Omega}$ holds for each $l_{1}, \ldots, l_{n} \in \mathbb{N}$. The sequence $\left(y_{k}\right)_{1}^{\infty} \subset R^{n} \backslash \bar{\Omega}$ runs through every point of

$$
M:=\left\{\left(x_{1}^{\left(l_{1}\right)}, \ldots, x_{n}^{\left(l_{n}\right)}\right): l_{1}, \ldots, l_{n} \in \mathbb{N}\right\} .
$$

Then the assertion of Theorem 1 holds with

$$
\underline{u}_{i}(x)=\sum_{k=1}^{l} \sum_{j=1}^{N} c_{k, j}^{(l)} \underline{E}_{j}\left(x-y_{k}\right) .
$$

Theorem 4. If the sequence of points $\left(y_{k}\right)_{1}^{\infty} \subset \dot{R}^{n} \backslash \bar{\Omega}$ is dense in an open set $U \subset R^{n} \backslash \bar{\Omega}$; then the assertion of Theorem 1 holds with

$$
\underline{u}_{l}(x)=\sum_{k=1}^{l} \sum_{j=1}^{N} c_{k, j}^{(l)} \underline{E}_{j}\left(x-y_{k}\right)
$$


The next theorem deals with the approximation by multipole potentials.

Theorem 5. Let $y_{1} \in R^{n} \backslash \bar{\Omega}$ be any point. Then the assertion of Theorem 1 holds with

$$
\underline{u}_{l}(x)=\sum_{|\alpha| \leq l} \sum_{j=1}^{N} c_{\alpha, j}^{(l)} D^{\alpha} \underline{E}_{j}\left(x-y_{1}\right) .
$$

In the next theorem the sequence $\left(y_{k}\right)_{1}^{\infty}$ lies dense on the boundary of a bounded domain $\Omega_{1} \supset \bar{\Omega}$. Then we need as supposition the unique solvability of a Dirichlet problem with respect to the exterior domain $R^{n} \backslash \bar{\Omega}_{1}$.

Theorem 6. Let $\Omega_{1} \supset \bar{\Omega}$ be a bounded domain with $\partial \Omega_{1}=\partial \bar{\Omega}_{1}$. We suppose that $\underline{L}^{*}$ has such a fundamental solution $\underline{E}^{*}$ that the Dirichlet problem

$$
\underline{L} \underline{v}=\underline{0} \quad \text { in } \quad R^{n} \backslash \bar{\Omega}_{1},\left.\quad D^{\alpha} v_{j}\right|_{a \Omega_{1}}=0 \quad \text { for } \quad|\alpha| \leq \tilde{t}_{j}-1(j=1, \ldots, m)
$$

has in the set of functions $\left\{\underline{v}=\underline{E}^{*} * \underline{g}: \underline{g} \in\left(D^{\prime}\left(R^{n}\right)\right)^{N}\right.$, supp $\left.\underline{\underline{ }} \subseteq \Gamma\right\}$ only the trivial solution. Here $\tilde{T}=\left(\tilde{t}_{1}, \ldots, \tilde{t}_{N}\right)$ is a multi-index with $0 \leq \tilde{t}_{j} \leq t$ and $\sum_{j=1}^{N} \tilde{t}_{j}=m$. If the sequence of points $\left(y_{k}\right)_{1}^{\infty} \subset R^{n} \backslash \bar{\Omega}$ is dense on $\partial \Omega_{1}$, then the assertion of Theorem 1 holds with

$$
\underline{u}_{l}(x)=\sum_{k=1}^{l} \sum_{j=1}^{N} \sum_{|\alpha| \leq \tilde{i}_{j}-1} c_{k, j, \alpha}^{(l)} D^{\alpha} \underline{E}_{j}\left(x-y_{k}\right) .
$$

Here $\underline{E}$ is the fundamental solution of $\underline{L}$ with $\underline{E}(x)=\left(\underline{E}^{*}(-x)\right)^{T}$.

\section{Preparation for the proof}

3.1 For an open set $\Omega_{0} \subseteq R^{n}$ and $0 \leq s<\infty$ we define

$$
\stackrel{\circ}{C} \cdot\left(\Omega_{0}\right)=\overline{C_{0}^{\infty}\left(\Omega_{0}\right)} \quad \text { (closure in the norm of } C^{\bullet}\left(\Omega_{0}\right) \text { ) }
$$

The elements of $\left(\stackrel{\circ}{C} \cdot\left(\Omega_{0}\right)\right)^{\prime}$ are distributions of order $s$ on $\Omega_{0}$. Obviously, for $f \in \check{C}^{\bullet} \cdot\left(\Omega_{0}\right)$ and $|\alpha| \leq s$ we have $\left.D^{\alpha} f\right|_{\partial \Omega_{0}}=0$. Denoting by $D_{n}$ the derivative on the smooth boundary $\Gamma=\partial \Omega$ in the direction of the exterior normal vector with respect to the domain $\Omega$, we can prove the following lemma (see [6]).

Lemma 3. It holds

$$
\stackrel{\circ}{C} \cdot\left(R^{n} \backslash \Gamma\right)=\left\{f \in \stackrel{\circ}{C} \cdot\left(R^{n}\right):\left.D_{n}^{j} f\right|_{\Gamma}=\grave{0} \text { for } j=0, \ldots, s\right\} .
$$

3.2 For the proof of the theorems we need the following lemmas.

Lemma 4. Suppose that $\underline{B}(x, D)$. is a Dirichlet matrix on $\Gamma$ of order $T=\left(t_{1}, \ldots, t_{N}\right)$, $t=t_{1} \geq \cdots \geq t_{N} \geq 0$ (see Definition 1), and $k_{0} \geq 0$ an integer. Then

(i) $B \in L\left(\stackrel{\circ}{C}^{k_{0}+T}\left(R^{n}\right), \prod_{s=1}^{t}\left(C^{k_{0}+t-s+1}(\Gamma)\right)^{N \prime}\right)$

(ii) $\underline{B}$ is a mapping from $C_{0}^{k_{0}+T}\left(R^{n}\right)$ onto $\prod_{s=1}^{t}\left(C^{k_{0}+t-o+1}(\Gamma)\right)^{N !}$. 
Here $\stackrel{\circ}{C}^{k_{0}+T}\left(R^{n}\right)=\stackrel{\circ}{C}^{k_{0}+t_{1}}\left(R^{n}\right) \times \ldots \times \stackrel{\circ}{C}^{k_{0}+t_{N}}\left(R^{n}\right)$. Furthermore $N$; is the number of those values of $j$ for which $t-t_{j} \leq s-1$. The assertion (ii) means that for each tuple $\dot{\varphi}=\left(\varphi^{1}, \ldots, \varphi^{t}\right), \varphi^{\prime}=\left(\varphi_{1}^{\prime}, \ldots, \varphi_{N_{0}^{\prime}}^{\prime}\right)^{T} \in\left(C^{k_{0}+t-s+1}(\Gamma)\right)^{N_{\bullet}^{\prime}}(s=1, \ldots, t)$, there exists always a vector function $\underline{u} \in C_{0}^{k_{0}+T}\left(R^{n}\right)$ with $\underline{B u}=\dot{\varphi}$, i.e. $\underline{B}^{1} \underline{u}=\varphi^{1}, \ldots, \underline{B^{t}} \underline{u}=\varphi^{t}$.

The following lemma is a consequence of Lemma 4 .

Lemma 5. Let $\underline{Q}(x, D)=\left(Q_{h j}(x, D)\right)_{\substack{h=1, \ldots, q \\ j=1, \ldots, N}}\left(Q_{h j}(x, D)=\sum_{|\alpha| \leq r_{h}+\ell_{j}} Q_{\alpha}^{h j}(x) D^{\alpha}, Q_{\alpha}^{h j} \in\right.$ $\left.C^{\infty}(\Gamma)\right)$ be $T$-normal. Here $R=\left(r_{1}, \ldots, r_{q}\right), r_{h} \leq-1$. Then

(i) $\underline{Q} \in L\left(\stackrel{\circ}{C}^{k_{0}+T}\left(R_{n}\right), C^{k_{0}-R}(\Gamma)\right)$

(ii) $\underline{Q}$ is a mapping from $C_{0}^{k_{0}+T}\left(R^{n}\right)$ onto $C^{k_{0}-R}(\Gamma)$, where $k_{0} \geq 0$ is an integer.

Now let $\left.\underline{L}\right|_{\Gamma}$ be an elliptic operator in the sense of Petrovskij as a boundary operator and $\left.D_{n}^{k} \underline{L}\right|_{\Gamma}=\left(\left.D_{n}^{k} L_{i j}\right|_{\Gamma}\right)_{i, j=1, \ldots, N}$, where $D_{n}$ is the exterior normal derivative on $\Gamma$. Furthermore let $\underline{b}=\left(\underline{b}_{1}, \ldots, \underline{b}_{m}\right)^{T}=\left(b_{h j}\right)_{\substack{n=1, \ldots, m \\ j=2, \ldots, N}}$ be a $T$-normal system of boundary operators on $\Gamma$. Then there holds

Lemma 6. The system $\left\{\underline{b}_{1}, \ldots, \underline{b}_{m},\left.\left.\underline{L}\right|_{\Gamma_{2}} D_{n} \underline{L}\right|_{\Gamma}, \ldots,\left.D_{n}^{k} \underline{L}\right|_{\mathrm{r}}\right\}$ is a $\tilde{T}$-normal system of matrices of boundary operators on $\Gamma$ with $\tilde{T}=T+k+1=\left(t_{1}+k+1, \ldots, t_{N}+k+1\right)$.

To prove this lemma we have to use the ellipticity of $\underline{L}$ in a suitable way.

3.3 If $\Omega_{0} \subseteq R^{n}$ is an open set, we denote by $W_{p}^{s}\left(\Omega_{0}\right)(s \geq 0$ integer, $1<p<\infty)$ the classical Sobolev spaces. Let $\dot{W}_{2}^{\prime}\left(\Omega_{0}\right)$ be the closure of $C_{0}^{\infty}\left(\Omega_{0}\right)$ in $W_{p}^{\prime}\left(\Omega_{0}\right)$. It holds $W_{p}^{\prime}\left(R^{n}\right)=\stackrel{\circ}{W}_{p}^{\prime}\left(R^{n}\right)$. Further let $W_{p^{\prime}}^{-\bullet}\left(\Omega_{0}\right)=\left(\stackrel{\circ}{W}_{p}^{\prime}\left(\Omega_{0}\right)\right)^{\prime}\left(s \geq 0, \quad p^{\prime}=\frac{p}{p-1}\right)$. For $-\infty<s<\infty$ and $1<p<\infty$ we define

$$
W_{p, \text { loc }}^{\prime}\left(R^{n}\right)=\left\{f \in D^{\prime}\left(R^{n}\right):\left.f\right|_{\Omega_{0}} \in W_{p}^{s}\left(\Omega_{0}\right) \text { for all bounded sets } \Omega_{0} \in R^{n}\right\} .
$$

The following assertion follows from well-known regularity theorems for elliptic operators.

Lemma 7. Let $\underline{L}(D)=\left(L_{i j}(D)\right)_{i, j=1, \ldots, N}$ be elliptic' in the sense of. Douglis-Nirenberg with $T=\left(t_{1}, \ldots, t_{N}\right)$ and $S=\left(s_{1}, \ldots, s_{N}\right)$. Furthermore let $E=\left(E_{i j}\right)_{i, j=1, \ldots, N}$ be a fundamental solution of LL. If $f \in W_{p}^{k_{0}-S}\left(R^{n}\right)\left(-\infty<k_{0}<\infty\right)$ is an element with $a$ compact support, then $\underline{E} * f \in W_{p, 10 c}^{k_{0}+T}\left(R^{n}\right)$.

3.4 A vector function $f$ is analytic on an open set $\Omega_{0} \subseteq R^{n}$, if for each point $y_{0} \in \Omega_{0}$ there exists a neighbourhood such that $f(x)$ can be represented as power series

$$
f(x)=\sum_{|\alpha|=0}^{\infty} \underline{d}_{\alpha}\left(x-y_{0}\right)^{\alpha}
$$

in this neighbourhood. If $D^{\alpha} f\left(y_{0}\right)=0$ for each $\alpha$ at any point $y_{0} \in \Omega_{0}$, we obtain $f=0$ in the connected component of $\Omega_{0}$ which contains $y_{0}$. Further the following lemma holds. 
Lemma 8. Let $f$ be an analytic vector function in a domain $\Omega_{0} \subseteq R^{\text {n }}$ and $y_{0}$ $=\left(y_{0,1}, \ldots, y_{0, n}\right) \in \Omega_{0}$ any point. Further for $i=1, \ldots, n$ let $\left(x_{i}^{(l)}\right)_{l=1}^{\infty} \subset R^{1^{*}}, x_{i}^{(l)} \neq y_{0, i}$ for all $l$, be a real sequence with yo,i as accumalation point, such that $\left(\dot{x}_{1}^{\left(l_{1}\right)}, \ldots, x_{n}^{\left(l_{n}\right)}\right) \in \Omega_{0}$ for all $l_{1}, \ldots, l_{n} \in \mathbb{N}$. If $\underline{f}\left(x_{1}^{\left(l_{1}\right)}, \ldots, x_{n}^{\left(l_{n}\right)}\right)=\underline{0}$ for $l_{1}, \ldots, l_{n} \in \mathbb{N}$, then $\underline{f}=\underline{0}$ in $\Omega_{0}$.

3.5 Now we investigate the unique solvability of the Cauchy problem. To do this we are firstly concerned with a statement about removability of singularities.

For a bounded set $A \subset R^{n}, \varepsilon>0$ and for real numbers $d$ with $0<d \leq n$ let

$$
H_{d}(A, \varepsilon):=\inf \left\{\sum_{k=1}^{\infty} r_{k}^{d}: A \subset \bigcup_{k=1}^{\infty} B_{x_{k}}^{r_{k}}, r_{k} \leq \varepsilon\right\}
$$

$\left(B_{x_{k}}^{r_{k}}:=\left\{x \in R^{n}:\left|x-x_{k}\right|<\varepsilon\right\}\right)$. The limit

$$
\lim _{\varepsilon \rightarrow 0} H_{d}(A, \varepsilon)=: H_{d}(A)
$$

is the $d$-dimensional Hausdorff measure of $A$. The $d$-dimensional Lebesgue measure coincides on compact subsets of a $d$-dimensional smooth submanifold of $R^{n}$ with $c_{d} H_{d}(A)$ where $c_{d}$ is a constant depending only on $d$.

The following lemma was proved by Harvey and Polking [9: Lemma 3.2].

Lemma 9. Suppose $K \subset R^{n}$ is compact. Given $\dot{k}<n$ and $\varepsilon>0$, there is a $\psi_{\varepsilon} \in C_{0}^{\infty}\left(R^{n}\right)$ with $\psi_{\varepsilon} \equiv 1$ in a neighbourhood of $K$ and supp $\psi_{\varepsilon} \subset K_{\varepsilon}=\left\{x \in R^{n}: d(x, K)<\varepsilon\right\}$ such that, for $|\alpha| \leq k$,

$$
\int_{R^{n}}\left|D^{\alpha} \psi_{e}(x)\right| d x \leq C_{\alpha} \varepsilon^{k-|\alpha|}\left(H_{n-k}(K)+\varepsilon\right),
$$

where $C_{\alpha}$ is independent of $\varepsilon$.

Lemma 10. Let $\Omega_{0} \subseteq R^{n}$ be an open set and $A$ a relatively closed subset of $\Omega_{0}$. Furthermore let $P(x, D)=\sum_{|\alpha| \leq k} a_{\alpha}(x) D^{\alpha}$ be any scalar linear differential operator with coefficients $a_{\alpha} \in C^{\infty}\left(\Omega_{0}\right)$. Suppose $k<n$ and $H_{n-k}(K)<\infty$ for each compact subset $K \subseteq A$. Then

$$
\lim _{e \rightarrow 0} \int f(x) P^{*}(x, D)\left(\varphi \psi_{e}\right)(x) d x=0
$$

for each $f \in C\left(\Omega_{0}\right)$ and for each $\varphi \in C_{0}^{\infty}\left(\Omega_{0}\right)$ with supp $\varphi \cap A \neq 0$.

Proof. The proof will be given in three steps.

Step 1. We write $P^{*}(x, D)=\sum_{|\alpha| \leq k} a_{\alpha}^{*}(x) D^{\alpha}$. Then we have

$$
\begin{aligned}
P^{*}(x, D)\left(\varphi \psi_{e}\right)(x) & =\sum_{|\alpha| \leq k} a_{\alpha}^{*}(x) D^{\alpha}\left(\varphi \psi_{e}\right)(x) \\
& =\sum_{|\alpha| \leq k} a_{\alpha}^{*}(x) \sum_{\beta \leq \alpha}\left(\begin{array}{l}
\alpha \\
\beta
\end{array}\right) D^{\alpha-\beta} \varphi(x) D^{\beta} \psi_{e}(x) \\
& =\sum_{|\beta| \leq k} D^{\beta} \psi_{\varepsilon}(x)\left(\sum_{\beta \leq \alpha,|\alpha| \leq k} a_{\alpha}^{*}(x)\left(\begin{array}{l}
\alpha \\
\beta
\end{array}\right) D^{\alpha-\beta} \varphi(x)\right) \\
& =\sum_{|\beta| \leq k} D^{\beta} \psi_{e}(x) \varphi^{\beta}(x)
\end{aligned}
$$


where $\varphi^{\beta}(x)=\sum_{\beta \leq \alpha,|\alpha| \leq k} a_{\alpha}^{*}(x)\left(\begin{array}{l}a \\ \beta\end{array}\right) D^{\alpha-\beta} \varphi(x)$. Let. $\varphi \in C_{0}^{\infty}\left(\Omega_{0}\right)$ be any function: Then we define $S=\operatorname{supp} \varphi$ and $K=S \cap A$. We get

$$
\begin{aligned}
\left|\int f(x) P^{*}(x, D)\left(\varphi \psi_{\varepsilon}\right)(x) d x\right| & \leq\|f\|_{C(S)} \int\left|P^{*}(x, D)\left(\varphi \psi_{e}\right)(x)\right| d x \\
& \leq\|f\|_{C(S)} \sum_{|\beta| \leq k} \int\left|D^{\beta} \psi_{e}(x) \| \varphi^{\beta}(x)\right| d x \\
& \leq\|f\|_{C(S)} \sum_{|\beta| \leq k}\left(\left\|\varphi^{\beta}\right\|_{C\left(R^{n}\right)} \int\left|D^{\beta} \psi_{e}(x)\right| d x\right) \\
& \leq C\|f\|_{C(S)} \sum_{|\beta| \leq k} \int\left|D^{\beta} \psi_{e}\right| d x \\
& \leq C\|f\|_{C(S)} \sum_{|\beta| \leq k} C_{\beta} \varepsilon^{k-|\beta|}\left(H_{n-k}(K)+\varepsilon\right) \\
& \leq C\|f\|_{C(S)}\left(H_{n-k}(K)+\varepsilon\right) .
\end{aligned}
$$

The constant $C$ is independent of $\varepsilon$.

Step 2. Now let $\Phi \in C^{\infty}\left(\Omega_{0}\right)$ be any function. Then we have

$$
\begin{aligned}
\left|\int \Phi(x) P^{*}\left(\varphi \psi_{e}\right)(x) d x\right| & =\left|\int P \Phi(x)\left(\varphi \psi_{e}\right)(x) d x\right| \\
& \leq\|\varphi P \Phi\|_{C(S)} \int\left|\psi_{\varepsilon}(x)\right| d x \\
& \leq\|\varphi P \Phi\|_{C(S)} C \varepsilon^{k}\left(H_{n-k}(K)+\varepsilon\right) .
\end{aligned}
$$

Therefore we get $\lim _{e \rightarrow 0} \int \Phi(x) P^{*}\left(\varphi \psi_{e}\right)(x) d x=0$ for each $\Phi \in C^{\infty}\left(\Omega_{0}\right)$.

Step 3. Let $\delta$ be any positive real number. For a given function $f \in C\left(\Omega_{0}\right)$ there exists a function $\Phi \in C_{0}^{\infty}\left(R^{n}\right)$ with $\|f-\Phi\|_{C(S)}<\delta\left(S=\operatorname{supp} \varphi, \varphi \in C_{0}^{\infty}\left(\Omega_{0}\right)\right)$. We get

$$
\begin{aligned}
\mid \int f(x) & P^{*}\left(\varphi \psi_{\varepsilon}\right)(x) d x \mid \\
& \leq\left|\int(f(x)-\Phi(x)) P^{*}\left(\varphi \psi_{e}\right)(x) d x\right|+\left|\int \Phi(x) P^{*}\left(\varphi \psi_{\varepsilon}\right)(x) d x\right| \\
& \leq\|f-\Phi\|_{C(S)} C\left(H_{n-k}(K)+\varepsilon\right)+\left|\int \Phi(x) P^{*}\left(\varphi \psi_{e}\right)(x) d x\right| .
\end{aligned}
$$

It follows

$$
\lim _{e \rightarrow 0}\left|\int f(x) P^{*}\left(\varphi \psi_{e}\right) d x\right| \leq \delta C H_{n-k}(K) .
$$

This holds for each $\delta>0$. Therefore we get $\lim _{e \rightarrow 0} \int f(x) P^{*}\left(\varphi \psi_{e}\right)(x) d x=0$ 【

Lemma 11. If $f \in C^{k-1}\left(\Omega_{0}\right)$ and $H_{n-1}(K)<\infty$ for each compact subset $K \subseteq \dot{A}$, then

$$
\lim _{e \rightarrow 0} \int f(x) P^{*}\left(\varphi \psi_{e}\right)(x) d x=0 .
$$

Proof. For suitable partial differential operators $Q_{\alpha}(x, D)$ with ord $Q_{\alpha} \leq 1$ we have $P(x, D)=\sum_{|\alpha| \leq k-1} Q_{\alpha}(x, D) D^{\alpha}$. Then

$$
\int f(x) P^{\bullet}\left(\varphi \psi_{e}\right)(x) d x=\sum_{|a| \leq k-1} \int D^{\alpha} f(x) Q_{\alpha}^{*}(x, D)\left(\varphi \psi_{e}\right)(x) d x .
$$

Here $D^{\alpha} f \in C\left(\Omega_{0}\right)$. Lemma 10 yields the assertion — 
Lemma 12. Let $\Omega_{0} \subseteq R^{n}$ be an open set and $A$ a relatively closed subset of $\Omega$. Suppose $H_{n-1}(K)<\infty$ for each compact subset $K \subseteq A$. Further let

$$
\underline{P}(x, D)=\left(P_{i j}(x, D)\right)_{i, j=1, \ldots, N}, \quad P_{i j}=\sum_{|\alpha| \leq s_{i}+t_{j}} a_{\alpha}^{i j}(x) D^{\alpha}, a_{\alpha}^{i j} \in C^{\infty}\left(\Omega_{0}\right)
$$

be a system of partial differential operators. Here $s_{i}$ and $t_{\text {; }}$ are integers with $t_{1} \geq t_{2} \geq$ $\ldots \geq t_{N} \geq 1$ and $0=s_{1} \geq s_{2} \geq \ldots \geq s_{N}$. If $\underline{u} \in C^{T-1}\left(\Omega_{0}\right)=C^{t_{1}-1}\left(\Omega_{0}\right) \times C^{t_{2}-1}\left(\Omega_{0}\right) \times$ $\cdots \times C^{t_{N}-1}\left(\Omega_{0}\right)$ satisfies $\underline{P} \underline{u}=\underline{0}$ in $\Omega_{0} \backslash A$, then $\underline{u}$ satisfies $\underline{P} \underline{u}=\underline{0}$ even in the whole set $\Omega_{0}$. (Then the set $A$ is said to be a removable singularity.)

Proof. We have to show

$$
\left\langle\varphi^{T}, \underline{P} \underline{u}\right\rangle=0 \quad\left(\underline{P} \underline{u} \in\left(D^{\prime}\left(\Omega_{0}\right)\right)^{N}\right) \text { for each } \underline{\varphi}=\left(\varphi_{1}, \ldots, \varphi_{N}\right)^{T} \in\left(C_{0}^{\infty}\left(\Omega_{0}\right)\right)^{N} .
$$

Let $\varphi$ be such a vector function. If $\operatorname{supp} \varphi \cap A=\emptyset$, then the assertion is clear. Suppose $\operatorname{supp} \varphi \cap A=: K \neq \emptyset$. Then with the $\psi_{\varepsilon}$ of Lemma 9 , we have

$$
\left\langle\underline{\varphi}^{T}, \underline{P} \underline{u}\right\rangle=\left\langle\left(\varphi \psi_{e}\right)^{T}, \underline{P} \underline{u}\right\rangle+\left\langle\left(\varphi\left(1-\psi_{e}\right)\right)^{T}, \underline{P} \underline{u}\right\rangle .
$$

Since $\psi_{e} \equiv 1$ in a neighbourhood of $K$ we have $\operatorname{supp}\left(\varphi\left(1-\psi_{e}\right)\right) \cap A=\emptyset$. Therefore $\left\langle\left(\varphi\left(1-\psi_{\varepsilon}\right)\right)^{T}, \underline{P} \underline{u}\right\rangle=0$ and

$$
\begin{aligned}
\left\langle\underline{\varphi}^{T}, \underline{P} \underline{u}\right\rangle=\left\langle\left(\underline{\varphi} \psi_{c}\right)^{T}, \underline{P} \underline{u}\right\rangle & =\sum_{i=1}^{N} \sum_{j=1}^{N}\left\langle\varphi_{i} \psi_{e}, P_{i j} u_{j}\right\rangle \\
& =\sum_{i=1}^{N} \sum_{j=1}^{N}\left\langle P_{i j}^{*}\left(\varphi_{i} \psi_{e}\right), u_{j}\right\rangle \\
& =\sum_{i=1}^{N} \sum_{j=1}^{N} \int u_{j}(x) P_{i j}^{*}\left(\varphi_{i} \psi_{e}\right)(x) d x .
\end{aligned}
$$

Here $u_{j} \in C^{t_{j}-1}\left(\Omega_{0}\right)$ and $P_{i j}$ is a differential operator with ord $P_{i j} \leq t_{j}$. Lemma 11 implies $\lim _{e \rightarrow 0}\left\langle P_{i j}^{*}\left(\varphi \psi_{\varepsilon}\right), u_{j}\right\rangle=0$. This proves the assertion

Lemma 12 is a generalization of [ 9 Theorem $4.3 /(b)]$ for systems.

Lemma 13. Let $\Omega_{0} \subseteq R^{n}$ be a domain and $K \subset \Omega_{0}$ a compact set with the following properties:

1. There is an open ball $B$, which can be divided by $K$ into two non-empty disjoint regions.

2. $H_{n-1}(K \cap B)<\infty$.

Further let $\underline{P}(x, D)=\left(P_{i j}(x, D)\right)_{i, j=1, \ldots, N}, P_{i j}(x, D)=\sum_{|a| \leq A_{i}+i_{j}} a_{\alpha}^{i j}(x) D^{\alpha}$, be elliptic in the sense of Douglis-Nirenberg. Suppose that the coefficients $a_{\alpha}^{i j}$ are analytic functions. Then the Cauchy problem

$$
\underline{P}_{\underline{u}}=\underline{Q} \text { in } \Omega_{0},\left.\quad D^{\alpha} u_{j}\right|_{K}=0 \text { for }|\alpha| \leq t_{j}-1 \quad(j=1, \ldots, N)
$$

has only the solution $\underline{u} \equiv \underline{Q}$ in $\Omega_{0}$. 
Proof. Let $\underline{u}$ be any solution of the Cauchy problem (4). In particular $\underline{P} \underline{u}=\underline{Q}$ in $B$ and $\left.D^{\alpha} u_{j}\right|_{K \cap B}=0$ for $|\alpha| \leq t_{j}-1, j=1, \ldots, N$. The both open sets we get, if one divides $B$ by $K$, are denoted by $\omega_{1}$ and $\omega_{2}$. We define a new vector function $\underline{v}$ on $B$ by

$$
\underline{v}=\left\{\begin{array}{lll}
\underline{u} & \text { in } & \omega_{1} \\
\underline{0} & \text { in } & B \backslash \omega_{1}=\omega_{2} \cup(K \cap B)
\end{array}\right.
$$

Obviously, $\underline{P} \underline{v}=Q$ in $\omega_{1} \cup \omega_{2}=B \backslash K .\left.D^{\alpha} u_{j}\right|_{K \cap B}=0$ for $|\alpha| \leq t_{j}-1(j=1, \ldots, N)$ implies $\underline{v} \in C^{T-1}(B)$. Considering $H_{n-1}(K \cap B)<\infty$ Lemma 12 yields that $K \cap B$ is a removable singularity. Hence, we have $\underline{P} \underline{v}=\underline{0}$ in $B$. Since $\underline{v} \equiv \underline{0}$ on the open set $\omega_{2}$ and since $\underline{v}$ is analytic on $B$ we get $\underline{v} \equiv \underline{0}$ on $B$. Therefore $\underline{u} \equiv \underline{Q}$ on the open set $\omega_{1}$. Since $\underline{u}$ is analytic on the domain $\Omega_{0}$ finally we have $\underline{u} \equiv \underline{0}$ on $\Omega_{0}$

3.6 The proofs of the approximation theorems are based on the following consequence of the Hahn-Banach theorem.

Lemma 14. Let $X$ be a normed space and let $X_{0}, X_{1}$ be linear subsets of $X$ with $X_{0} \subset X_{1}$. If every $F \in X^{\prime}$ with $\left\langle f_{0}, F\right\rangle=0$ for all $f_{0} \in X_{0}$ also satisfies the equation $\left\langle f_{1}, F\right\rangle=0$ for all $f_{1} \in X_{1}$, then $\bar{X}_{0} \supseteq X_{1}$.

\section{Proof of the theorems}

We will represent the proof of Theorem 1 in detail. To prove the other theorems we have to modify this proof only slightly.

4.1 Proof of Theorem 1. The proof will be given in 14 steps.

Step 1. First we show that the approximability of an element $\underline{f} \in \prod_{h=1}^{m} D_{h}(\Gamma)$ implies $\left\langle\underline{f}^{T}, \underline{c}^{*} \underline{v}\right\rangle=0$ for all $\underline{v} \in N^{*}$. Let $\left(\underline{u}_{1}\right)_{1}^{\infty}$,

$$
\underline{u}_{l}(x)=\sum_{k=1}^{l} \sum_{j=1}^{N} \sum_{|\alpha| \leq t-1} c_{k, j, \alpha}^{(l)} D^{\alpha} \underline{E}_{j}\left(x-y_{k}\right),
$$

be a sequence with

$$
\lim _{h \rightarrow \infty} \sum_{h=1}^{m}\left\|f_{h}-\underline{b}_{h} \underline{u}_{h}\right\|_{D_{h}(\Gamma)}=0
$$

We have $\left.\underline{u}_{1}\right|_{\Omega} \in\left(C^{\infty}(\bar{\Omega})\right)^{N}$ and $\underline{L} \underline{u}_{1}=\underline{Q}$ in $\Omega$ for all $l$. Since $\underline{L}^{*} \underline{v}=\underline{Q}$ in $\Omega$ and $\underline{b}^{*} \underline{v}=\underline{0}$ on $\Gamma$ for $\underline{v} \in N^{*}$ we get from Green's formula

$$
0=\sum_{h=1}^{m}\left\langle\underline{b}_{h} \underline{u}_{h}, \underline{c}_{h}^{*} \underline{v}\right\rangle=\left\langle\left(\underline{b} \underline{u}_{h}\right)^{T}, \underline{c^{*}} \underline{v}\right\rangle
$$

for all $l$ and all $\underline{v} \in N^{*}$. From this, relation (5) and inclusion $\underline{c}^{*} \underline{v} \in\left(\prod_{h=1}^{m} D_{h}(\Gamma)\right)^{\prime}$ one $\operatorname{gets}\left\langle\underline{f}^{T}, \underline{c^{*}} \underline{v}\right\rangle=0$.

Step 2. In the following we are concerned with the reverse inclusion. Let $\underline{F}=$ $\left(F_{1}, \ldots, F_{m}\right)^{T} \in \prod_{h=1}^{m}\left(D_{h}(\Gamma)\right)^{\prime}$ be any continuous and linear functional with

$$
\cdot \sum_{h=1}^{m}\left(\underline{b}_{h}\left(D^{\alpha} \underline{E}_{j}\left(x-y_{k}\right)\right), F_{h}(x)\right\rangle=\left\langle\left(\underline{b}\left(D^{\alpha} \underline{E}_{j}\left(x-y_{k}\right)\right)\right)^{T}, \underline{F}(x)\right\rangle=0
$$


for $k \in \mathbb{N},|\alpha| \leq t-1$, and $j=1, \ldots, N$. This yields

$$
\left\langle\left(\underline{b}\left(D^{\alpha} \underline{E}\left(x-y_{k}\right)\right)\right)^{T}, \underline{F}(x)\right\rangle=\underline{0}
$$

for $|\alpha| \leq t-1$ and $k \in \mathbb{N}$. We will show that for this $\underline{F}$ the equation $\left\langle\underline{f}^{T}, \underline{F}\right\rangle=0$ holds for those $\underline{f} \in \prod_{h=1}^{m} D_{h}(\Gamma)$ which satisfy the condition $\left\langle\underline{f}^{T}, \underline{c}^{*} \underline{v}\right\rangle=0$ for all $\underline{v} \in N^{*}$. By means of Lemma 14 one can get then the approximability of these $f$ by finite linear combinations of $\underline{b}\left(D^{\alpha} \underline{E}_{j}\left(x-y_{k}\right)\right)(k \in \mathbb{N},|\alpha| \leq t-1, j=1, \ldots, N)$.

Step 3. Let $k_{0} \geq-1$ be so large that $C^{k_{0}-r_{h}}(\Gamma) \subset D_{h}(\Gamma)$ for $h=1, \ldots, m$ and that these imbeddings are continuous. This implies

$$
\prod_{h=1}^{m}\left(D_{h}(\Gamma)\right)^{\prime} \subset \prod_{h=1}^{m}\left(C^{k_{0}-r_{h}}(\Gamma)\right)^{\prime}=\left(C^{k_{0}-R}(\Gamma)\right)^{\prime}
$$

and the continuity of this imbedding. Here $R=\left(r_{1}, \ldots, r_{m}\right)$, and the $r_{h}$ are such negative integers that ord $b_{h j} \leq r_{h}+t_{j}$ for $j=1, \ldots, N$. We can consider $E$ as an element of $\left(C^{k_{0}-R}(\Gamma)\right)^{\prime}$. Lemma 4 implies the inclusion

$$
\underline{b} \in L\left(\stackrel{\circ}{C}^{k_{0}}+T\left(R^{n}\right), C^{k_{0}-R}(\Gamma)\right)
$$

Hence, the dual operator $\underline{b}^{\prime}$ belongs to $L\left(\left(C^{k_{0}-R}(\Gamma)\right)^{\prime},\left(\stackrel{\circ}{C}^{k_{0}+T}\left(R^{n}\right)\right)^{\prime}\right)$ and $\underline{b^{\prime}} \underline{F}$ to $\left(\stackrel{\circ}{C}^{k_{0}+T}\left(R_{n}\right)\right)^{\prime}$. Furthermore we get $\left\langle\underline{\varphi}^{T}, \underline{b^{\prime}} \underline{F}\right\rangle=\left\langle(\underline{b} \varphi)^{T}, \underline{F}\right\rangle=0$ for each $\varphi \in\left(C_{0}^{\infty}\left(R^{n} \backslash \Gamma\right)\right)^{N}$. This implies supp $\underline{b^{\prime}} \underline{F} \subseteq \Gamma$.

Step 4. We define $d_{0}:=\inf \{|x-y|: x \in \Gamma, y \in K\}$ and $d_{1}:=\sup \{|x-y|: x \in$ $\Gamma, y \in K\}$. We suppose that the subsequence $\left(y_{k}^{\prime}\right)_{1}^{\infty}$ of $\left(y_{k}\right)_{1}^{\infty}$ consists of those points $y_{k}$, for which the inequalities

$$
\frac{1}{2} d_{0}<\inf \left\{\left|x-y_{k}\right|: x \in \Gamma\right\} \quad \text { and } \quad \sup \left\{\left|x-\dot{y}_{k}\right|: x \in \Gamma\right\}<2 d_{1}
$$

hold. Obviously, each point of $K$ is again an accumulation point of this subsequence. Let $\eta_{0} \in C_{0}^{\infty}\left(R^{n}\right)$ be an odd function with

$$
\eta_{0}(x)=1 \text { for } \frac{1}{4} d_{0}<|x|<4 d_{1} \quad \text { and } \eta_{0}(x)=0 \text { for }|x|<\frac{1}{8} d_{0} .
$$

We have $\eta_{0} E_{i j} \in C_{0}^{\infty}\left(R^{n}\right)(i, j=1, \ldots, N)$ and $\eta_{0} \underline{E}\left(x-y_{k}^{\prime}\right)=\underline{E}\left(x-y_{k}^{\prime}\right)$ for each $k \in \mathbb{N}$ and for each $x$ from a neighbourhood of $\Gamma$. This implies

$$
\underline{b}\left(\left(D^{\alpha}\left(\eta_{0} \underline{E}\right)\right)\left(x-y_{k}^{\prime}\right)\right)=\underline{b}\left(\left(D^{\alpha} \underline{E}\right)\left(\dot{x}-y_{k}^{\prime}\right)\right)
$$

for each $x \in \Gamma, k \in \mathbb{N}$ and for each $\alpha$. From (7) we obtain

$$
\left\langle\left(\underline{b}\left(D^{\alpha}\left(\eta_{0} \underline{E}\right)\left(x-y_{k}^{\prime}\right)\right)\right)^{T}, \underline{F}(x)\right\rangle=\left\langle\left(D^{\alpha}\left(\eta_{0} \underline{E}\right)\left(x-\dot{y}_{k}^{\prime}\right)\right)^{T},\left(\underline{b^{\prime}} \underline{F}\right)(x)\right\rangle=\underline{0}
$$

for $k \in \mathbb{N}$ and for each $\alpha$ with $|\alpha| \leq t-1$.

Step. 5. Since $\underline{b}^{\prime} E \in\left(\stackrel{\circ}{C}^{k_{0}}+T\left(R^{n} .\right)\right)^{\prime}$ has a compact support the convolution $\underline{E}^{*} *\left(\underline{b}^{\prime} \underline{F}\right)$ exists. The imbedding $W_{p}^{k_{0}+1+T}\left(R^{n}\right) \subset \dot{C}^{k_{0}+T}\left(R^{n}\right)$ is continuous for $p>n$. Consequently, we have

$$
\left(\stackrel{\circ}{C}^{k_{0}+T}\left(R^{n}\right)\right)^{\prime} \subset W_{p^{\prime}}^{-k_{0}-1-T}\left(R^{n}\right)\left(p^{\prime}=\frac{p}{p-1}\right) \quad \text { and } \quad \underline{b^{\prime}} \underline{F} \in W_{p^{\prime}}^{-k_{0}-1-T}\left(R^{n}\right) .
$$


Because $\underline{L}$ is elliptic in the sense of Petrovskij, i.e. $T=\left(t_{1}, \ldots, t_{N}\right)$ and $S=(0, \ldots, 0)$; $\underline{L}^{*}$ is elliptic in the sense of Douglis-Nirenberg with $T^{*}=(t, t, \ldots, t)$ and $S^{*}=\left(t_{1}-t, t_{2}-\right.$ $\left.t, \ldots, t_{N}-t\right)=T-t$. Since $-k_{0}-1-T=-k_{0}+t-S^{*}$ we can write $\underline{b^{\prime}} E \in W_{p^{\prime}}^{-k_{0}-1-t-S^{*}}\left(R^{n}\right)$. Applying Lemma 7 we get

$$
\underline{E}^{*} *\left(\underline{b}^{\prime} \underline{F}\right) \in W_{p^{\prime}, l o c}^{-k_{0}-1-t+T^{*}}\left(R^{n}\right)=\left(W_{p^{\prime}, l o c}^{-k_{0}-1}\left(R^{n}\right)\right)^{N} .
$$

From $\underline{L}^{*}\left(\underline{E}^{*} *\left(\underline{b}^{\prime} \underline{F}\right)\right)=\underline{b^{\prime}} \underline{F}$ and $\operatorname{supp} \underline{b}^{\prime} \underline{F} \subseteq \Gamma$ we get $\underline{L}^{*}\left(\underline{E}^{*} *\left(\underline{b^{\prime}} \underline{F}\right)\right)=\underline{0}$ in $R^{n} \backslash \Gamma$ and consequently $\underline{E}^{*} *\left(\underline{b}^{\prime} \underline{F}\right) \in\left(C^{\infty}\left(R^{n} \backslash \Gamma\right)\right)^{N^{*}}$. Since $\underline{L}^{*}$ is à elliptic differential operator with constant coefficients $\underline{E}^{*} *\left(\underline{b}^{\prime} \underline{F}\right)$ is even analytic in $R^{n} \backslash \Gamma$.

Step 6. We have

$$
\underline{E}^{*} *\left(\underline{b^{\prime}} \underline{F}\right)=\left(\left(1-\eta_{0}\right) \underline{E}^{*}\right) *\left(\underline{b}^{\prime} \underline{F}\right)+\left(\eta_{0} \underline{E}^{*}\right) *\left(\underline{b}^{\prime} \underline{F}\right)
$$

Since

$$
\left(\eta_{0} \underline{E}^{*}\right) *\left(\underline{b}^{\prime} \underline{F}\right) \in\left(C_{0}^{\infty}\left(R^{n}\right)\right)^{N} \quad \text { and } \quad \underline{E}^{*} *\left(\underline{b^{\prime}} \underline{E}\right) \in\left(C^{\infty}\left(R^{n} \backslash \Gamma\right)\right)^{N}
$$

we get $\left(\left(1-\eta_{0}\right) \underline{E}^{*}\right) *\left(\underline{b}^{\prime} \underline{F}\right) \in\left(C^{\infty}\left(R^{n} \backslash \Gamma\right)\right)^{N}$. We have

$$
\begin{aligned}
\operatorname{supp}\left(\left(1-\eta_{0}\right) \underline{E}^{*}\right) *\left(\underline{b}^{\prime} \underline{F}\right) & \subseteq \operatorname{supp}\left(\underline{b^{\prime}} \underline{F}\right)+\operatorname{supp}\left(\left(1-\eta_{0}\right) \underline{E}^{*}\right) \\
& \subseteq \Gamma+\operatorname{supp}\left(1-\eta_{0}\right) \\
& \subseteq\left\{x+z: x \in \Gamma,|z| \leq \frac{1}{4} d_{0} \text { or }|z| \geq 4 d_{1}\right\} .
\end{aligned}
$$

Hence for each $y$ satifying $\frac{1}{2} d_{0}<\inf \{|x-y|: x \in \Gamma\} \leq \sup \{|x-y|: x \in \Gamma\}<2 d_{1}$ one gets the assertion $\left(\left(\left(1-\eta_{0}\right) \underline{E}^{*}\right) *\left(\underline{b^{\prime}} \underline{F}\right)\right)(y)=\underline{0}$ and therefore

$$
D^{\alpha}\left(\left(\left(1-\eta_{0}\right) \underline{E}^{*}\right) *\left(\underline{b^{\prime}} \underline{F}\right)\right)\left(y_{k}^{\prime}\right)=\underline{0} \quad \text { for each } \alpha \text { and } k \in \mathbb{N} \text {. }
$$

Hence we have $D^{\alpha}\left(\underline{E}^{*} *\left(\underline{b^{\prime}} \underline{F}\right)\right)\left(y_{k}^{\prime}\right)=D^{\alpha}\left(\left(\eta_{0} \underline{E}^{*}\right) *\left(\underline{b^{\prime}} \underline{F}\right)\right)\left(y_{k}^{\prime}\right)$ for each $\alpha$ and $k \in \mathbb{N}$.

Step 7. Considering $\eta_{0} E_{i j} \in C_{0}^{\infty}\left(R^{n}\right), \underline{E}^{*}(x)=(\underline{E}(-x))^{T}, \eta_{0}(x)=\eta_{0}(-x)$, and (8) we have, for $|\alpha| \leq t-1$,

$$
\begin{aligned}
D^{\alpha}\left(\underline{E}^{*} *\left(\underline{b}^{\prime} \underline{F}\right)\right)\left(y_{k}^{\prime}\right) & =D^{\alpha}\left(\left(\eta_{0} \underline{E}^{*}\right) *\left(\underline{b^{\prime}} \underline{F}\right)\right)\left(y_{k}^{\prime}\right) \\
& =\left(\left(D^{\alpha}\left(\eta_{0} \underline{E}^{*}\right)\right) *\left(\underline{b^{\prime}} \underline{F}\right)\right)\left(y_{k}^{\prime}\right) \\
& =\left\langle D^{\alpha}\left(\eta_{0} \underline{E}^{*}\right)\left(y_{k}^{\prime}-x\right),\left(\underline{b^{\prime}} \underline{F}\right)(x)\right\rangle \\
& =(-1)^{|\alpha|}\left(\left(D^{\alpha}\left(\eta_{0} \underline{E}\right)\left(x-y_{k}^{\prime}\right)\right)^{T},\left(\underline{b}^{\prime} \underline{E}\right)(x)\right)=\underline{0} .
\end{aligned}
$$

Consequently, $D^{\alpha}\left(\underline{E}^{*} *\left(\underline{b}^{\prime} \underline{F}\right)\right)\left(y_{k}^{\prime}\right)=\underline{0}$ for all $k$ and for each $\alpha$ with $|\alpha| \leq t-1$.

Because $\underline{E}^{*} *\left(\underline{b^{\prime}} \underline{F}\right)$ is infinitely differentiable in a neighbourhood of $K$ and because the sequence $\left(y_{k}^{\prime}\right)_{1}^{\infty}$ is dense in $K$ it follows

$$
\left.D^{\alpha}\left(\underline{E}^{*} *\left(\underline{b^{\prime}} \underline{F}\right)\right)\right|_{K}=\underline{0}
$$

for each $\alpha$ with $|\alpha| \leq t-1$. Furthermore $\underline{L}^{*}\left(\underline{E}^{*} *\left(\underline{b}^{\prime} \underline{F}\right)\right)=\underline{0}$ holds in the connected set $R^{n} \backslash \bar{\Omega}$. Lemma 13 yields that the Cauchy problem

$$
\underline{L} \underline{v}=\underline{0} \quad \text { in } R^{n} \backslash \bar{\Omega},\left.\quad D^{\alpha} \underline{v}\right|_{K}=\underline{0} \text { for }|\alpha| \leq t-1
$$


has only the solution $\underline{v}=\underline{0}$. ( $\underline{L}^{*}$ is elliptic in the sense of Douglis-Nirenberg with $t_{i}^{*}=$ $\cdots t_{N}^{*}=t$ and $s_{i}^{*}=t_{i}-t$.) Consequently, we get $\underline{E}^{*} *\left(\underline{b}^{\prime} \underline{F}\right) \equiv \underline{0}$ on $R^{n} \backslash \bar{\Omega}$. Thus, we have

$$
\underline{E}^{*} *\left(\underline{b}^{\prime} \underline{F}\right) \in\left(W_{p^{\prime}, l o c}^{-k_{0}-1}\left(R^{n}\right)\right)^{N} \text { and } \operatorname{supp}\left(\underline{E}^{*} *\left(\underline{b^{\prime}} \underline{F}\right)\right) \subseteq \bar{\Omega} \text {. }
$$

One can easily conclude $\underline{E}^{*} *\left(\underline{b}^{\prime} \underline{F}\right) \in\left(\left(\stackrel{\circ}{C}^{k_{0}+1}\left(R^{n}\right)\right)^{N}\right)^{\prime}$. From this and from supp $\left(\underline{E}^{*} *\right.$ $\left.\left(\underline{b}^{\prime} \underline{F}\right)\right) \subseteq \bar{\Omega}$ we obtain

$$
\left\langle\underline{f}^{T}, \underline{E}^{*} *\left(\underline{b}^{\prime} \underline{F}\right)\right\rangle=0
$$

for all $\underline{f} \in\left(\stackrel{\circ}{C}^{k_{0}+1}\left(R^{n}\right)\right)^{N}$ with $\left.\underline{f}\right|_{R^{n} \backslash \bar{\Omega}} \in\left(\stackrel{\circ}{C}^{k_{0}+1}\left(R^{n} \backslash \bar{\Omega}\right)\right)^{N}$ and $\left.\underline{f}\right|_{\Omega}=\underline{0}$.

Step 8. In the following $f \perp N^{*}$ means that $\sum_{i=1}^{N} \int_{\Omega} f_{i} v_{i} d x=0$ for all $\underline{y}=\left(v_{1}, \ldots, v_{N}\right)^{T}$ $\in N^{*}$. Now let $\varphi \in\left(C_{0}^{\infty}\left(R^{n} \backslash \Gamma\right)\right)^{N}$ be any vector function with $\varphi_{1}:=\varphi \ln \perp N^{*}$. Then $\varphi_{1} \in\left(C_{0}^{\infty}(\Omega)\right)^{N}$ and $\varphi_{2}:=\left.\varphi\right|_{R^{n}} \backslash \bar{\Omega} \in\left(C_{0}^{\infty}\left(R^{n} \backslash \bar{\Omega}\right)\right)^{N}$. We consider the boundary value problem

$$
\underline{L} \underline{u}=\varphi_{1} \text { in } \Omega, \quad \underline{b} \underline{u}=\underline{0} \text {, on } \Gamma \text {. }
$$

Because of the requirement $\varphi_{1} \perp N^{*}$ this problem has a solution $\underline{u}_{1} \in\left(C^{\infty}(\bar{\Omega})\right)^{N}(\mathrm{~s}$. Lemma 2). In particular we have

$$
\underline{b} \underline{u}_{1}=\underline{0} \text {. }
$$

We extend $\underline{u}_{1}$ to a vector function $\underline{\tilde{u}}_{1} \in C_{0}^{k_{0}+1+T}\left(R^{n}\right)$ and define $\tilde{\varphi}_{1}:=\underline{L} \underline{\tilde{u}}_{1}$ $\in\left(C_{0}^{k_{0}+1}\left(R^{n}\right)\right)^{N}$. It is $\underline{\underline{w}}_{1} \in C^{k_{0}+1-R}(\Gamma)$. Equation (10) yields

$$
\underline{b} \underline{\tilde{u}}_{1}=\underline{0} \text {. }
$$

Furthermore $\tilde{\varphi}_{1}$ is an extension of $\underline{\varphi}_{1}$. For this reason $D_{n}^{j} \tilde{\varphi}_{1}=\underline{0}$ holds for $j=0, \ldots, k_{0}+1$ $\left(D_{n}\right.$ the exterior normal derivative on $\Gamma$ ). From Lemma 3 one gets $\tilde{\varphi}_{1} \in\left(\stackrel{\circ}{C}^{k_{0}+1}\left(R^{n} \backslash \Gamma\right)\right)^{N}$, which implies $\left.\tilde{\varphi}_{1}\right|_{R^{n} \backslash \bar{\Omega}} \in\left(\stackrel{\circ}{C}^{k_{0}+1}\left(R^{n} \backslash \bar{\Omega}\right)\right)^{N}$.

We split up $\varphi=\left(\varphi-\tilde{\varphi}_{1}\right)+\tilde{\varphi}_{1}$. Obviously, $\left(\varphi-\tilde{\varphi}_{1}\right) \in\left(\stackrel{\circ}{C}^{k_{0}+1}\left(R^{n}\right)\right)^{N},\left.\left(\varphi-\tilde{\varphi}_{1}\right)\right|_{R^{n} \backslash \bar{\Omega}} \epsilon$ $\left(\stackrel{\circ}{C}^{k_{0}+1}\left(R^{n} \backslash \bar{\Omega}\right)^{N}\right.$ and $\left.\left(\varphi-\tilde{\varphi}_{1}\right)\right|_{\Omega}=\varphi_{1}-\varphi_{1}=0$. From $(9)$ we follow $\left\langle\left(\varphi-\tilde{\varphi}_{1}\right)^{T}, \underline{E}^{*} *\left(\underline{b^{\prime}} \underline{F}\right)\right\rangle=$ 0 . Considering (11) we get

$$
\begin{aligned}
\left\langle\varphi^{T}, \underline{E}^{*} *\left(\underline{b}^{\prime} \underline{F}\right)\right\rangle & =\left\langle\underline{\tilde{e}}_{1}^{T}, \underline{E}^{*} *\left(\underline{b}^{\prime} \underline{F}\right)\right\rangle=\left\langle\left(\underline{L} \underline{\tilde{u}}_{1}\right)^{T}, \underline{E}^{*} *\left(\underline{b}^{\prime} \underline{F}\right)\right\rangle \\
& =\left\langle\underline{\tilde{u}}_{1}^{T}, \underline{L}^{*}\left(\underline{E}^{*} *\left(\underline{b}^{\prime} \underline{F}\right)\right)\right\rangle=\left\langle\underline{\tilde{u}}_{1}^{T},\left(\underline{b}^{\prime} \underline{F}\right)\right\rangle=\left\langle\left(\underline{b} \underline{\tilde{u}}_{1}\right)^{T}, \underline{F}\right\rangle=0 .
\end{aligned}
$$

Thus we have $\left\langle\underline{\varphi}^{T}, \underline{E}^{*} *\left(\underline{b}^{\prime} \underline{F}\right)\right\rangle=0$ for those $\varphi \in\left(C_{0}^{\infty}\left(R^{n} \backslash \Gamma\right)\right)^{N}$ which have the property $\left.\varphi\right|_{0} \perp N^{*}$. From this one can conclude (cf. [6: p.296]) $\left(\underline{f}^{T}, \underline{E}^{*} *\left(\underline{b^{\prime}} \underline{F}\right)\right\rangle=0$ for all $\dot{f} \in$ $\left(\stackrel{\circ}{C}^{k_{0}+1}\left(R^{n} \backslash \Gamma\right)\right)^{N}$ with $\left.\underline{f}\right|_{\Omega} \perp N^{*}$.

Step 9. We define the operator $\underline{R}_{k_{0}+1}$ by the equation

$$
\underline{R}_{k_{0}+1} g=\left\{\left.\underline{g}\right|_{\Gamma},\left.D_{n} \underline{g}\right|_{\Gamma}, \ldots,\left.D_{n}^{k_{0}+1} \underline{g}\right|_{\Gamma}\right\}
$$

for $g \in\left(\dot{C}^{k_{0}+1}\left(R^{n}\right)\right)^{N}\left(D_{n}\right.$ the exterior normal derivative). Using the fact

$$
\left(\stackrel{\circ}{C}^{k_{0}+1}\left(R^{n} \backslash \Gamma\right)\right)^{N}=\left\{\underline{g} \in\left(\stackrel{\circ}{C}^{k_{0}+1}\left(R^{n}\right)\right)^{N}: \underline{R}_{k_{0}+1} \underline{g}=\dot{0}\right\}
$$


(Lemma 3) we have

$$
\left\langle\underline{f}^{T}, \underline{E}^{*} *\left(\underline{b}^{\prime} \underline{F}\right)\right\rangle=0
$$

for all $\underline{f} \in\left(\stackrel{\circ}{C}^{k_{0}+1}\left(R^{n}\right)\right)^{N}$ with $\underline{R}_{k_{0}+1} \underline{f}=\underline{0}$ and $\left.\underline{f}\right|_{\Omega} \perp N^{*}$.

Step 10. Now we want to investigate the behaviour of the distribution $\underline{E}^{*} *\left(\underline{b^{\prime}} \underline{F}\right)$ on an arbitrary vector function from $\left(\stackrel{\circ}{C}^{k_{0}+1}\left(R^{n}\right)\right)^{N}$. We define the operator $A=\left\{\underline{R}_{k_{0}+1}, P\right\}$ for $f \in\left(\stackrel{\circ}{C}^{k_{0}+1}\left(R^{n}\right)\right)^{N}$ by

$$
A \underline{f}:=\left\{\underline{R}_{k_{0}+1} \underline{f}, \sum_{k=1}^{r^{*}}\left(\left.\underline{f}\right|_{\Omega}, \underline{v}^{(k)}\right) \underline{v}^{(k)}\right\} \in\left(\prod_{j=0}^{k_{0}+1}\left(C^{k_{0}+1-j}(\Gamma)\right)^{N}\right) \times N^{*}=: X
$$

Here $\left\{\underline{v}^{(1)}, \ldots, \underline{v}^{\left({ }^{*}\right)}\right\}$ is a basis in $\cdot N^{*}$ and $\left(\left.\underline{f}\right|_{\Omega}, \underline{v}^{(k)}\right)=\sum_{i=1}^{N} \int_{\Omega} f_{i} v_{i}^{(k)} d x$ for all elements $\underline{v}^{(k)}=\left(v_{1}^{(k)}, \ldots, v_{N}^{(k)}\right)^{T}$. Then (12) means $\underline{E}^{*} *\left(\underline{b}^{\prime} E\right) \in(\operatorname{Ker} A)^{\perp}$,

$$
(\operatorname{Ker} A)^{\perp}:=\left\{\underline{H} \in\left(\left(\stackrel{\circ}{C}^{k_{0}+1}\left(R^{n}\right)\right)^{N}\right)^{\prime}:\left\langle\underline{f}^{T}, \underline{H}\right\rangle=0 \forall \underline{f} \in\left(\stackrel{\circ}{C}^{k_{0}+1}\left(R^{n}\right)\right)^{N}, A \underline{f}=\dot{0}\right\} .
$$

$X$ is a Banach space with respect to the norm

$$
\begin{gathered}
\|\{\underline{g}, \underline{v}\}\|_{X}=\sum_{j=0}^{k_{0}+1}\left\|\underline{g}_{j}\right\|_{C^{k_{0}+1-j}(\Gamma)}+\sum_{k=1}^{\boldsymbol{r}^{*}}\left|d_{k}\right| \\
\left(\underline{\dot{g}}:=\left\{\underline{g}_{j}\right\}_{0}^{k_{0}+1} \in \prod_{j=0}^{k_{0}+1}\left(C^{k_{0}+1-j}(\Gamma)\right)^{N}, \quad \underline{v}:=\sum_{k=1}^{\dot{r}^{*}} d_{k} \underline{v}^{(k)} \in N^{*}\right) .
\end{gathered}
$$

Obviously, we have $A \in L\left(\left(\stackrel{\circ}{C}^{k_{0}+1}\left(R^{n}\right)\right)^{N}, X\right)$.

Now we show $\operatorname{Im} A=X$. Let $\underline{\dot{g}}=\left\{\underline{g}_{j}\right\}_{0}^{k_{0}+1} \in \prod_{j=0}^{k_{0}+1}\left(C^{k_{0}+1-j}(\Gamma)\right)^{N}$ be any element and $\underline{v}=\sum_{k=1}^{r^{*}} d_{k} \underline{v}^{(k)}$ any vector function from $N^{*}$. We look for a vector function $g \in\left(\dot{C}^{k_{0}+1}\right.$ $\left.\left(R^{n}\right)\right)^{N}$ with $\underline{R}_{t_{0}+1} \underline{g}=\underline{\dot{g}}$ and $P g=\underline{v}$. Lemma $5 /($ ii) implies the existence of a vector function $\underline{\tilde{g}} \in\left(C_{0}^{k_{0}+1}\left(R^{n}\right)\right)^{N}$ with $\underline{R}_{k_{0}+1} \underline{\tilde{g}}=\underline{\dot{g}}$. Let $\left\{\underline{\psi}^{(j)}\right\}_{1}^{r^{*}} \subset\left(C_{0}^{\infty}(\Omega)\right)^{N}$ be a biorthogonal system to $\left\{\underline{v}^{(k)}\right\}_{1}^{r^{*}}$, i.e.,

$$
\left(\underline{\psi}^{(j)}, \underline{v}^{(k)}\right)=\sum_{i=1}^{N} \int_{\Omega} \psi_{i}^{(j)} v_{i}^{(k)} d \dot{x}=\left\{\begin{array}{llll}
1 & \text { for } & k=j \\
0 & \text { for } & k \neq j
\end{array} \quad\left(k, j=1, \ldots, r^{*}\right) .\right.
$$

We set

$$
\underline{g}:=\tilde{g}+\sum_{j=1}^{r^{*}}\left(d_{j}-\left(\tilde{g} \mid \Omega, \underline{v}^{(j)}\right)\right) \psi^{(j)}
$$

Since $\underline{R}_{k_{0}+1} \underline{\psi}^{(j)}=\underline{\dot{0}}$ we have $\underline{R}_{k_{0}+1} \underline{g}=\underline{R}_{k_{0}+1} \tilde{g}=\dot{g}$. Furthermore, $\left(\left.\underline{g}\right|_{\Omega}, \underline{v}_{k}\right) \dot{=} d_{k}$ implies $P \underline{q}=\underline{v}$. Therefore we have shown $\operatorname{Im} A=X$.

Step 11. Since $\operatorname{Im} A$ is closed the Closed Range Theorem implies $\operatorname{Im} A^{\prime}=(\operatorname{Ker} A)^{\perp}$. Since $\operatorname{Im} A=X$ we get, using a corollary of the Open Mapping Theorem and the Closed Range Theorem (see [16: p.147]), $\left(A^{\prime}\right)^{-1} \in L\left(\operatorname{Im} A^{\prime}, X^{\prime}\right)$. . Consequently $\left(A^{\prime}\right)^{-1} \in$ $L\left((\operatorname{Ker} A)^{\perp}, X^{\prime}\right)$. Because of $\underline{E}^{*} *\left(\underline{b^{\prime}} \underline{F}\right) \in(\operatorname{Ker} A)^{\perp}$ the equation

$$
A^{\prime} \underline{\Lambda}=\underline{E}^{*} *\left(\underline{b^{\prime}} \underline{F}\right)
$$


has a unique solution

$$
\underline{\Lambda}=\left\{\underline{\Lambda}_{1}, \underline{\Lambda}_{2}\right\} \in\left(\prod_{j=0}^{k_{0}+1}\left(C^{k_{0}+1-j}(\Gamma)\right)^{N}\right)^{\prime} \times\left(N^{*}\right)^{\prime}
$$

Therefore we have

$$
\begin{aligned}
\left\langle\underline{f}^{T}, \underline{E}^{*} *\left(\underline{b}^{\prime} \underline{E}\right)\right\rangle & =\left\langle\underline{f}^{T}, A^{\prime} \underline{\Lambda}\right\rangle \\
& =\left\langle(A \underline{f})^{T}, \underline{\Lambda}\right\rangle \\
& =\left\langle\left(\underline{R}_{k_{0}+1} \underline{f}\right)^{T}, \underline{\Lambda}_{1}\right\rangle+\left\langle P \underline{f}, \underline{\Lambda}_{2}\right\rangle \\
\cdots & =\sum_{j=0}^{k_{0}+1}\left\langle\left(D_{n}^{j} \underline{f} \mid \Gamma\right)^{T}, \underline{\lambda}_{j}\right\rangle+\left\langle\sum_{k=1}^{r^{*}}\left(\left.\underline{f}\right|_{\Omega}, \underline{v}^{(k)}\right) \underline{v}^{(k)}, \underline{\Lambda}_{2}\right\rangle
\end{aligned}
$$

and then

$$
\left\langle\underline{f}^{T}, \underline{E}^{*} *\left(\underline{b}^{\prime} \underline{F}\right)\right\rangle=\sum_{j=0}^{k_{0}+1}\left\langle\left(D_{n}^{j} \underline{f} \mid \Gamma\right)^{T}, \underline{\lambda}_{j}\right\rangle+\sum_{i=1}^{N} \int_{\Omega} f_{i} v_{i} d x
$$

for all elements $\underline{f} \in\left(\stackrel{\circ}{C}^{k_{0}+1}\left(R^{n}\right)\right)^{N}$ with $\underline{\Lambda}_{1}=\left\{\underline{\lambda}_{j}\right\}_{0}^{k_{0}+1} \in \prod_{j=0}^{k_{0}+1}\left(\left(C^{k_{0}+1-j}(\Gamma)\right)^{N}\right)^{\prime}$ and all $\underline{v}=\sum_{k=1}^{r^{*}}\left\langle\underline{v}^{(k)}, \underline{\Lambda}_{2}\right) \underline{v}^{(k)} \in N^{*}$.

Step 12. Now let $\underline{u} \in C_{0}^{k_{0}+1+T}\left(R^{n}\right)$ be any vector function. Then $\underline{L} \underline{u} \in\left(C_{0}^{k_{0}+1}\left(R^{n}\right)\right)^{N}$. We set $\underline{f}:=\underline{L} \underline{u}$ into (13). Then one gets

$$
\left\langle(\underline{L} \underline{u})^{T}, \underline{E}^{*} *\left(\underline{b^{\prime}} \underline{F}\right)\right\rangle=\sum_{j=0}^{k_{0}+1}\left\langle\left(D_{n}^{j} \underline{L} \underline{u} \mid \Gamma\right)^{T}, \underline{\lambda}_{j}\right\rangle+\sum_{i=1}^{N} \int_{\mathbf{\Omega}}(\underline{L} \underline{u})_{i} v_{i} d x .
$$

From Green's formula (Lemma 1)' we obtain $\left(\underline{L}^{*} \underline{v}=\underline{0}, \underline{b} \underline{v}^{*}=\underline{0}\right.$ !)

$$
\sum_{i=1}^{N} \int_{\Omega}(\underline{L} \underline{u})_{i} v_{i} d x \fallingdotseq-\sum_{h=1}^{m}\left(\underline{b}_{h} \underline{\underline{u}}, \underline{c}_{h}^{*} \underline{v}\right)=:-\left\langle(\underline{b} \underline{u})^{T}, \underline{c^{*}} \underline{v}\right\rangle
$$

Since

$$
\left\langle(\underline{L} \underline{u})^{T}, \underline{E}^{*} *\left(\underline{b}^{\prime} \underline{F}\right)\right\rangle=\left\langle\underline{u}^{T}, \underline{L}^{*}\left(\underline{E}^{*} *\left(\underline{b}^{\prime} \underline{F}\right)\right)\right\rangle=\left\langle\underline{u}^{T}, \underline{b}^{\prime} \underline{F}\right\rangle=\left\langle(\underline{b} \underline{u})^{T}, \underline{F}\right\rangle
$$

we have

$$
\left\langle(\underline{b} \underline{u})^{T}, \underline{F}\right\rangle=\sum_{j=0}^{k_{0}+1}\left\langle\left(D_{n}^{j} \underline{L} \underline{u} \mid \Gamma\right)^{T}, \underline{\lambda}_{j}\right\rangle-\left\langle(\underline{b} \underline{u})^{T}, \underline{c}^{*} \underline{v}\right\rangle
$$

for all $\underline{u} \in C_{0}^{k_{0}+1+T}\left(R^{n}\right)$ with a certain vector function $\underline{v}$ from $N^{*}$.

Step 13. Now we show $\underline{\lambda}_{j}=\underline{0}$ for $j=0, \ldots, k_{0}+1$. From Lemma 6 we get the normality of $\left\{\underline{b}_{1}, \ldots, \underline{b}_{m},\left.\underline{L}\right|_{\Gamma},\left.D_{n} \underline{L}\right|_{\Gamma}, \ldots,\left.D_{n}^{k_{0}+1} \underline{L}\right|_{\Gamma}\right\}$. Let $j_{0}$ be any integer with $0 \leq j_{0} \leq$ $k_{0}+1$ and $g \in\left(C^{k_{0}+1-j_{0}}(\Gamma)\right)^{N}$ any vector function. Lemma $5 /(\mathrm{ii})$ implies the existence of a vector function $\underline{u} \in C_{0}^{k_{0}+1+T}\left(R^{n}\right)$ with

$$
\underline{b} \underline{u}=\underline{0}, \quad,\left.\quad D_{n}^{j} \underline{L} \underline{u}\right|_{\Gamma}=\underline{0} \quad \text { for } j \neq j_{0}, 0 \leq j \leq k_{0}+1
$$

and

$$
\left.D_{n}^{j o} \underline{L} \underline{u}\right|_{\Gamma}=\underline{q}
$$


If we apply (14) to this function $\underline{u}$, we get $\left\langle\underline{g}^{T}, \underline{\lambda}_{j 0}\right\rangle=0$, which implies $\underline{\lambda}_{j 0}=\underline{0}$. Since this holds for all $j_{0}=0, \ldots, k_{0}+1$, we, obtain

$$
\left\langle(\underline{b} \underline{u})^{T}, \underline{F}\right\rangle=-\left\langle(\underline{b} \underline{u})^{T}, \underline{c}^{*} \underline{v}\right\rangle
$$

for all $\underline{u} \in C_{0}^{k_{0}+1+T}\left(R^{n}\right)$.

Step 14. Let $\underline{f} \in \prod_{h=1}^{m} D_{h}(\Gamma)$ be any element with

$$
\left(\underline{f}^{T}, \underline{c}^{*} \underline{v}\right)=0
$$

for all $v \in N^{*}$. The condition $(\mathrm{a} 2)$ for the spaces $D_{h}(\Gamma)(h=1, \ldots, m)$ implies the existence of a sequence $\left(\varphi_{1}\right)_{1}^{\infty} \subset \prod_{h=1}^{m} C^{\infty}(\Gamma)$ with $\lim _{l \rightarrow \infty}\left\|f-\varphi_{l}\right\|_{\prod_{h=1}^{m} D_{h}(\Gamma)}=0$. Using Lemma $5 /\left(\right.$ ii) we can conclude that for every $l$ there exists a vector function $\underline{u}^{(l)} \in$ $C_{0}^{k_{0}+1+T}\left(R^{n}\right)$ with $\underline{b}^{(l)}=\underline{\varphi}_{1}$. From $(15)$ it follows $\left\langle\left(\varphi_{1}\right)^{T}, \underline{F}\right)=-\left\langle\left(\varphi_{1}\right)^{T}, \underline{c}^{*} \underline{v}\right\rangle$ for all $l$ and therefore $\lim _{4 \rightarrow \infty}\left\langle\left(\varphi_{1}\right)^{T}, \underline{F}\right)^{\prime}=-\lim _{4 \rightarrow \infty}\left\langle\left(\varphi_{1}\right)^{T}, \underline{c}^{*} \underline{v}\right\rangle$. Since $E$ and $\underline{c}^{*} \underline{v}$ are elements of $\left(\prod_{h=1}^{m} D_{h}(\Gamma)\right)^{\prime}$ and because of $(16)$ we get finally the desired equation $\left\langle\underline{f}^{T}, E\right\rangle=0$

4.2 Proof of the Theorems 2-6. The proofs of the six theorems differ essentially only in the way how to get $\underline{E}^{*}\left(\underline{b^{\prime}} \underline{F}\right) \equiv \underline{0}$ in $R^{n} \backslash \bar{\Omega}$. This result we obtained in the proof of Theorem 1 in Step 7. Furthermore there are modifications with respect to the requirement (6) on $\underline{F}$. Therefore we only want to describe firstly how to replace the requirement (6) and secondly how to get $\underline{E}^{*} *\left(\underline{b^{\prime}} \underline{F}\right) \equiv \underline{0}$ in $R^{n} \backslash \bar{\Omega}$ in Step 7 of the proof.

Proof of Theorem 2. 1. $F$ is a functional with

$$
\left\langle\left(\underline{b}\left(D^{\alpha} \underline{E}_{j}\left(x-y_{k}\right)\right)\right)^{T}, \underline{F}(x)\right\rangle=0
$$

for $k \in \mathbb{N},|\alpha| \leq \tilde{t}_{j}-1, j=1, \ldots, N$. 2. In Step 7 of the proof we get

$$
\left.D^{\alpha}\left(\underline{E}^{*} *\left(\underline{b^{\prime}} \underline{F}\right)\right)_{j}\right|_{\partial U}=\dot{0}
$$

for $|\alpha| \leq \tilde{t}_{j}-1, j=1, \ldots, N .\left(\underline{E}^{*} *\left(\underline{b^{\prime}} \underline{F}\right)\right)_{j}$ is the j-th component of $\underline{E}^{*} *\left(\underline{b^{\prime}} \underline{F}\right)$.) Furthermore we have $\underline{L}^{*}\left(\underline{E}^{*} *\left(\underline{b}^{\prime} \underline{F}\right)\right)=\underline{0}$ in $U$. Since we have required the unique solvability of the Dirichlet problem we get $\underline{E}^{*} *\left(\underline{b^{\prime}} \underline{E}\right) \equiv \underline{Q}$ in $U \subset R^{n} \backslash \bar{\Omega}$. Because $\underline{E}^{*} *\left(\underline{b}^{\prime} \underline{F}\right)$ is analytic in the connected open set $R^{n} \mid \bar{\Omega}$ one obtains $\underline{E}^{*}\left(\underline{b}^{\prime} \underline{F}\right) \equiv \underline{0}$ in $R^{n} \backslash \bar{\Omega}$.

Proof of Theorem 3.' 1: $E$ is a functional with

$$
\left(\left(\underline{b}\left(\underline{E}_{j}\left(x-y_{k}\right)\right)\right)^{T}, \underline{F}(x)\right)=0
$$

for $k \in \mathbb{N}$ and $j=1, \ldots, N:$ : : One gets

$$
\left(\underline{E}^{* *} *\left(\underline{b^{\prime}} \underline{E}\right)\right)\left(\dot{y}_{k}^{\prime}\right)=\underline{0}
$$

for all $k$. Lemma 8 implies $\underline{E}^{*} *\left(\underline{b^{\prime}} \underline{F}\right) \equiv 0$ in $R^{n} \backslash \bar{\Omega}$.

Proof of Theorem 4. 1. $E$ is a functional with

$$
\left(\left(\underline{b}\left(\underline{E}_{j}\left(x-y_{k}\right)\right)\right)^{T}, E(x)\right)=0
$$


for $k \in \mathbb{N}$ and $j=1, \ldots, N$. 2. One gets $\left(\underline{E}^{*} \underline{b}^{\prime} \underline{F}\right) \equiv \underline{0}$ on the open set $U \subset R^{n} \backslash \bar{\Omega}$. The analytizity of $\underline{E}^{*}\left(\underline{b}^{\prime} \underline{F}\right)$ implies $\underline{E}^{*} *\left(\underline{b}^{\prime} \underline{F}\right) \equiv \underline{0}$ in $R^{n} \backslash \bar{\Omega}$.

Proof of Theorem 5. 1. $E$ is a functional with

$$
\left\langle\left(\underline{b}\left(D^{\alpha} \underline{E}_{j}\left(x-y_{1}\right)\right)\right)^{T}, \underline{F}(x)\right\rangle=0
$$

for all $\alpha$. 2. One gets $D^{\alpha}\left(\underline{E}^{*} *\left(\underline{b^{\prime}} \underline{F}\right)\right)\left(y_{1}\right)=\underline{0}$ for all $\alpha$ and then $\underline{E}^{*} *\left(\underline{b^{\prime}} \underline{F}\right) \equiv \underline{Q}$ in $R^{n} \backslash \bar{\Omega}$.

Proof of Theorem 6. 1. $E$ is a functional with

$$
\left\langle\left(\underline{b}\left(D^{\alpha} \underline{E}_{j}\left(x-y_{k}\right)\right)\right)^{T}, \underline{F}(x)\right\rangle=0
$$

for $k \in \mathbb{N},|\alpha| \leq \tilde{t}_{j}-1, j=1, \ldots, N$. 2. One gets $\left.D^{\alpha}\left(\underline{E^{*} *}(\underline{b} \underline{F})\right)_{j}\right|_{\text {an }}=0$ for $|\alpha| \leq \tilde{t}_{j}-1, j=1, \ldots, N$. The suppositions of Theorem 6 yield $\underline{E}^{*} *(\underline{b} \underline{E}) \equiv \underline{0}$ in $R^{n} \backslash \bar{\Omega}_{1}$ and by this also in $R^{n} \backslash \bar{\Omega}$.

\section{REFERENCES}

[1] BeckerT, H.: Eine bemerkenswerte Eigenschaft der Lösungen des Dirichletschen Problems bei linearen elliptischen Differentialgleichungen. Math. Ann. 139 (1960), 255 264.

[2] Beyer, K.: Approximation durch Lösungen elliptischer Randwertprobleme. Rostock. Math. Kolloq. 26 (1984), 27 - 34.

[3] BeYER, K.: Zur Approximation durch Multipolpotentiale der Laméschen Gleichungen. Rostock. Math. Kolloq. 40 (1990), 29 - 34.

[4] FrEedEn, W. and R. REUTER: A constructive method for solving the displacement boundary value problem of elastostatics by use of global basis systems. Heidelberg Scientific Center TR, 89.03.007, 1989.

[5] GöpferT, A .: Über L L Approximationssätze - eine Eigenschaft der Lösungen elliptischer Differentialgleichungen: Math. Nachr. 31 (1966), 1 - 24.

[6] Нама N, U.: Approximation durch Lösungen elliptischer Randwertprobleme auf geschlossenen Hyperflächen. Math. Nachr. 136 (1988), 285 - 301.

[7] Hamann, U.: Approximation mittels Linearkombinationen von Fundamentallösungen elliptischer Differentialoperatoren. Math. Nachr. 154 (1991), 265 - 284.

[8] НАмАN N, U.: Approximation by linear combinations of fundamental solution of the Lamé operator. Math. Nachr. 164 (1993), 271 : 282.

[9] HaRvey, R. and J.C. Polking: Removable singularities of linear partial differential equations. Acta Math. 125 (1970), 39 - 56.

[10] Kupradze, V.D.: Three-Dimensional Problems in the Theory of Elasticity and Thermoelasticity (in Russian). Moscow: Nauka 1976. 
[11] Rojtberg, I. and Y.A. RojtBeRg: On approximations the solutions of the elliptic boundary value problem by linear combinations of fundamental solution (in Russian. Dokl. Acad. Nauk Ukrain. 12 (1992), 15 - 20.

[12] Rojtberg, Y.A. and Z.G. ShefTel: $A$ theorem on homeomorphisms for elliptic systems and its applications (in Russian). Mat. Sbornik 78(120) (1969), 446 - 472.

[13] ROJTBERg, Y.A. and Z.G. SHEFTEL: On the density of the solutions of elliptic boundary value problems in the sense of Petrovskij systems in functional spaces on manifolds. In: Symposium "Analysis on Manifolds with Singularities", Breitenbrunn 1990 (TeubnerTexte zur Mathematik: Vol. 131)(eds.: B.-W. Schulze and H. Triebel). Stuttgart-Leipzig 1992, pp. $176 \cdot 180$.

[14] Scrulze, B.-W. and G. Wildenhain: Methoden der Potentialtheorie für elliptische Differentialgleichungen beliebiger Ordnung. Berlin: Akademie-Verlag 1977, and BaselStuttgart: Birkhn auser Verlag 1977.

[15] Wildengain, G.: Approximation in Sobolev-Räumen durch Lösungen allgemeiner elliptischer Randwertprobleme bei Gleichungen beliebiger Ordnung. Rostock. Math. Kolloq. 22 (1983), $43-56$.

[16] W LокA, J.: Funktionalanalysis und Anwendungen. Berlin- New York: Walter de Gruyter 1971.

Received 05.04.1993; in revised form 14.09.1993 